\title{
DISSECTING THE GRAVITATIONAL LENS B1608+656. I. LENS POTENTIAL RECONSTRUCTION*
}

\author{
S. H. Suyu ${ }^{1,2,3}$, P. J. Marshall ${ }^{4}$, R. D. Blandford ${ }^{1,2}$, C. D. Fassnacht $^{5}$, L. V. E. Koopmans ${ }^{6}$, J. P. McKean ${ }^{5,7}$, and \\ T. TrEu ${ }^{4,8}$ \\ ${ }^{1}$ Theoretical Astrophysics, 103-33, California Institute of Technology, Pasadena, CA, 91125, USA; suyu@astro.uni-bonn.de \\ ${ }^{2}$ Kavli Institute for Particle Astrophysics and Cosmology, Stanford University, PO Box 20450, MS 29, Stanford, CA 94309, USA \\ ${ }_{3}^{3}$ Argelander Institut für Astronomie, Auf dem Hügel 71, D-53121 Bonn, Germany \\ ${ }^{4}$ Department of Physics, University of California, Santa Barbara, CA 93106-9530, USA \\ ${ }^{5}$ Department of Physics, University of California at Davis, 1 Shields Avenue, Davis, CA 95616, USA \\ ${ }^{6}$ Kapteyn Astronomical Institute, University of Groningen, P.O. Box 800, 9700AV Groningen, The Netherlands \\ ${ }^{7}$ Max-Planck-Institut für Radioastronomie, Auf dem Hügel 69, D-53121 Bonn, Germany \\ Received 2008 April 16; accepted 2008 September 23; published 2009 January 9
}

\begin{abstract}
Strong gravitational lensing is a powerful technique for probing galaxy mass distributions and for measuring cosmological parameters. Lens systems with extended source-intensity distributions are particularly useful for this purpose since they provide additional constraints on the lens potential (mass distribution). We present a pixelated approach to modeling the lens potential and source-intensity distribution simultaneously. The method makes iterative and perturbative corrections to an initial potential model. For systems with sources of sufficient extent such that the separate lensed images are connected by intensity measurements, the accuracy in the reconstructed potential is solely limited by the quality of the data. We apply this potential reconstruction technique to deep Hubble Space Telescope observations of B1608+656, a four-image gravitational lens system formed by a pair of interacting lens galaxies. We present a comprehensive Bayesian analysis of the system that takes into account the extended source-intensity distribution, dust extinction, and the interacting lens galaxies. Our approach allows us to compare various models of the components of the lens system, which include the point-spread function (PSF), dust, lens galaxy light, source-intensity distribution, and lens potential. Using optimal combinations of the PSF, dust, and lens galaxy light models, we successfully reconstruct both the lens potential and the extended source-intensity distribution of B1608+656. The resulting reconstruction can be used as the basis of a measurement of the Hubble constant. As an illustration of the astrophysical applications of our method, we use our reconstruction of the gravitational potential to study the relative distribution of mass and light in the lensing galaxies. We find that the mass-to-light ratio for the primary lens galaxy is $(2.0 \pm 0.2) h M_{\odot} L_{\mathrm{B}, \odot}^{-1}$ within the Einstein radius $\left(3.9 \mathrm{~h}^{-1} \mathrm{kpc}\right)$, in agreement with what is found for noninteracting lens galaxies at the same scales.

Key words: black hole physics - galaxies: nuclei - gravitational waves - relativity
\end{abstract}

\section{INTRODUCTION}

Strong gravitational lens systems provide a tool for probing galaxy mass distributions (independent of their light profiles) and for measuring cosmological parameters (e.g., Kochanek et al. 2006, and references therein). Lens systems with extended source-intensity distributions are of special interest because they provide additional constraints on the lens potential (and hence the surface mass density) due to surface brightness conservation. In this case, simultaneous determination of the source-intensity distribution and the lens potential is needed. To describe either the source-intensity or the lens potential/mass distribution, there are two approaches in the literature: (1) "parametric," or better, "simply parameterized," using simple, physically motivated functional forms described by a few $(\sim 10)$ parameters (e.g., Kochanek; 1991; Kneib et al.; 1996; Keeton 2001; Marshall 2006; Jullo et al. 2007), and (2) pixel-based ("pixelated," or "free-form," or sometimes, inaccurately, "nonparametric") modeling on a grid, which has been done for both the source intensity (e.g., Wallington et al. 1996; Warren \& Dye 2003;

\footnotetext{
* Based in part on observations made with the NASA/ESA Hubble Space Telescope, obtained at the Space Telescope Science Institute, which is operated by the Association of Universities for Research in Astronomy, Inc., under NASA contract NAS 5-26555. These observations are associated with program GO-10158.

8 Sloan Fellow, Packard Fellow.
}

Treu \& Koopmans 2004; Dye \& Warren 2005; Koopmans 2005; Brewer \& Lewis 2006; Suyu et al. 2006; Wayth \& Webster 2006; Dye et al. 2008) and the lens potential/mass distribution (e.g., Williams \& Saha 2000; Bradač et al. 2005; Koopmans 2005; Saha et al. 2006; Suyu \& Blandford 2006; Jee et al. 2007; Vegetti $\&$ Koopmans 2008). Most of the developed lens modeling methods are simply parameterized. In particular, for the measurement of the Hubble constant, lens potential/mass models prior to Saha et al. (2006) have been simply parameterized because most of the strong lens systems with time delay measurements have only point sources (as opposed to extended sources) to constrain the lens potential/mass distribution. A precise measurement of the value of $H_{0}$ is important for testing the flat $\Lambda$-cold dark matter (CDM) model and studying dark energy. The cosmic microwave background (CMB) allows determination of cosmological parameters with high accuracy with the exception of $H_{0}$ (e.g., Komatsu et al. 2008). An independent measurement of $H_{0}$ to better than a few percent precision provides the single most useful complement to the CMB for dark energy studies (Hu 2005).

Koopmans (2005) developed a method for pixelated source intensity and lens potential reconstruction that is based on the potential correction scheme proposed by Blandford et al. (2001). This pixelated potential reconstruction method is applicable to lens systems with extended source-intensity distributions. Pixelbased modeling has the advantage over simply-parameterized modeling in the flexibility in the parametrization. This is 
especially important in complex lens systems (e.g., multicomponent source galaxies or multiple lens galaxies) where simplyparameterized models may become inadequate. Furthermore, pixel-based modeling has the capabilities of detecting dark matter substructures (Koopmans 2005; Vegetti \& Koopmans 2008).

In this paper, we present a lens modeling technique that is similar to that of Koopmans (2005), but in a Bayesian framework to allow quantitative comparison between various source intensity and lens potential models. The point-spread function (PSF), lens galaxy light, and dust models are also incorporated in this scheme. Therefore, this method provides a way to rank these data models (with the five interdependent components: source-intensity distribution, lens potential, PSF, lens galaxy light, and dust) quantitatively. There are also propagation effects due to structures along the line of sight (LOS), but we ignore this for now and characterize this in a forthcoming paper (Paper II).

We choose to reconstruct the lens potential instead of the surface mass density because (1) it is the quantity that directly relates to the cosmological parameters via the time delays and angular diameter distance ratios and (2) the surface mass density can, in principle, be easily obtained by differentiation. In contrast, Williams \& Saha (2000) and Saha et al. (2006) pixelized the surface mass density. Since the surface mass density over the entire lens plane is required in the integral for obtaining the lens potential, the conversion of the (finite) gridded mass density to the lens potential is not straightforward.

We apply the pixelated potential reconstruction method to B1608+656 (Myers et al. 1995), a quadruple image gravitational lens system with an extended source at $z_{\mathrm{s}}=1.394$ (Fassnacht et al. 1996), and two interacting galaxy lenses at $z_{\mathrm{d}}=0.6304$ (Myers et al. 1995). B1608+656 is special in that it is the only four-image gravitational lens systems with all three independent time delays between the images measured with errors of only a few percent (Fassnacht et al. 1999, 2002). Thus, it provides a great opportunity to measure the Hubble constant, which is the subject of Paper II. To obtain the Hubble constant to high precision, an accurate lens potential model is crucial. Koopmans \& Fassnacht (1999) modeled this system using simply parameterized lens potentials, but did not account for the presence of dust and the extended source intensity. Koopmans et al. (2003) improved on the simply parameterized modeling of the lens potential with the treatment of dust, the use of a simply parameterized extended source-intensity distribution, and the inclusion of constraints from stellar dynamics. However, Suyu \& Blandford (2006) showed that this most up-to-date simply parameterized lens model in Koopmans et al. (2003) fails certain tests such as the crossing of the critical curve through the saddle point of the figure-eight-shaped intensity contour of the merging images. This suggests that the pixelated potential method may be better suited than a simply parameterized method for the two interacting galaxies. In this paper, we deliver a comprehensive analysis of the B1608+656 system that incorporates the effects of the extended source intensity, presence of dust, and interacting lenses. The dissection of B1608+656 allows us to study the relative distribution of mass and light in the interacting lens galaxies.

The outline of the paper is as follows. In Section 2, we introduce the pixelated potential reconstruction method. We demonstrate the method using simulated data in Section 3 and generalize the method to real data in Section 4. The remaining sections of the paper target B1608+656. In Section 5, we summarize the Hubble Space Telescope (HST) observations of
B1608+656 and present the image processing. In Section 6, we show a pixelated potential reconstruction of B1608+656. Finally, in Section 7, we comment on the mass-to-light (M/L) ratio in B1608+656 based on the results of our lensing analysis. In Paper II, we use the resulting potential reconstruction of B1608+656 together with a study of the lens environment to infer the value of the Hubble constant.

Throughout this paper, we assume a flat $\Lambda$-CDM universe with $\Omega_{m}=0.26, \Omega_{\Lambda}=0.74$, and $H_{0}=100 \mathrm{~h} \mathrm{~km} \mathrm{~s}^{-1} \mathrm{Mpc}^{-1}$ (Komatsu et al. 2008). For the lens and source redshifts in B1608+656, $1^{\prime \prime}$ on the sky corresponds to $4.9 h^{-1} \mathrm{kpc}$ on the lens plane and $6.1 \mathrm{~h}^{-1} \mathrm{kpc}$ on the source plane.

\section{PIXELATED POTENTIAL RECONSTRUCTION}

In the following subsections, we present the pixelated potential reconstruction method. Section 2.1 contains the formalism of the method, and Section 2.2 is a practical implementation of the method.

\subsection{Formalism for Iterative and Perturbative Potential Corrections}

The iterative and perturbative potential correction scheme for lens systems with extended sources was first suggested by Blandford et al. (2001) and studied by Koopmans (2005), Suyu \& Blandford (2006), and recently by Vegetti \& Koopmans (2008). The pixelated potential reconstruction method that we present here is similar to that in Koopmans (2005) but differs in the numerical details and our use of Bayesian analysis, which allows for model comparison. The method in Vegetti \& Koopmans (2008) is also based on Bayesian analysis and has adaptive gridding on the source plane. In the rest of the section, we briefly outline the theory of pixelated potential reconstruction.

The central concept for this method is to start with an initial lens potential model and to correct it, perturbatively and iteratively, to obtain an estimate of the true lens potential. The initial lens potential will usually be simply parameterized (to allow faster convergence with a smaller number of parameters) and ideally would be close to the true potential. It will then be refined via corrections on a grid of pixels. Obtaining the parameter values in the initial lens potential is often a nonlinear process; in contrast, the potential correction in each iteration is a linear inversion.

One way to think about this procedure is to observe that in a perfectly observed image, nested intensity contours in the source plane map onto multiple regions of the image plane. Intensity is preserved by the lens and so the map is from a set of single source contours to the corresponding image contours. The only freedom that we have is to slide image points along the contours. Using the fact that the deflection field is curlfree effectively removes this freedom. What we describe is a procedure to determine this map that takes into account a finite PSF, dust extinction, and source-intensity contamination by the lens galaxy light. In Paper II, we also include the influence of propagation effects.

To keep the formalism simple for the moment, let us ignore the effects of the PSF, dust extinction, and lens galaxy light. Let $\vec{\theta}$ be the coordinates on the image plane and $\vec{\beta}$ be the coordinates on the source plane. Let $I_{\mathrm{d}}(\vec{\theta})$ be the observed image intensity of a lensed extended source, and let $\psi(\vec{\theta})$ be an initial scaled 
surface potential model ${ }^{9}$ for the lens system. Given $\psi(\vec{\theta})$, one can obtain the best-fitting source-intensity distribution (e.g., Suyu et al. 2006, and references therein). Let $I_{\mathrm{s}}(\vec{\theta}(\vec{\beta}))$ be the source intensity translated to the image plane via the potential model, $\psi(\vec{\theta})$, where $\vec{\theta}$ and $\vec{\beta}$ are related via the lens equation $\vec{\theta}=\vec{\beta}-\vec{\nabla} \psi(\vec{\theta})$. We define the intensity deficit (also known as the image residual) on the image plane by

$$
\delta I(\vec{\theta})=I_{\mathrm{d}}(\vec{\theta})-I_{\mathrm{s}}(\vec{\theta}(\vec{\beta})) .
$$

Suppose the initial lens potential model is perturbed from the true potential, $\psi_{0}(\vec{\theta})$, by $\delta \psi(\vec{\theta})$ :

$$
\psi(\vec{\theta})=\psi_{0}(\vec{\theta})+\delta \psi(\vec{\theta})
$$

For a given image (fixed $I_{\mathrm{d}}(\vec{\theta})$ ) and the initial potential model $\psi(\vec{\theta})$, we can relate the intensity deficit to the potential perturbation $\delta \psi(\vec{\theta})$ by

$$
\delta I(\vec{\theta})=\frac{\partial I_{\mathrm{s}}(\vec{\beta})}{\partial \vec{\beta}} \cdot \frac{\partial \delta \psi(\vec{\theta})}{\partial \vec{\theta}},
$$

to first order in $\delta \psi(\vec{\theta})$ (see e.g., Suyu \& Blandford 2006 for details). The source-intensity gradient $\partial I_{\mathrm{s}}(\vec{\beta}) / \partial \vec{\beta}$ implicitly depends on the potential model $\psi(\vec{\theta})$ since the source position $\vec{\beta}$ (where the gradient is evaluated) is related to $\psi(\vec{\theta})$ via the lens equation. We can solve Equation (3) for $\delta \psi(\vec{\theta})$ given the intensity deficit and source-intensity gradients, update the initial (or previous iteration's) potential model, and repeat the process of source-intensity reconstruction and potential correction until the potential converges to the true solution with zero intensity deficit. In Section 2.2, we focus on solving Equation (3).

\subsection{Implementation of Pixelated Potential Reconstruction}

\subsubsection{Probability Theory}

The first step in solving Equation (3) for the potential perturbation is to obtain the source-intensity gradients and the intensity deficit, which appear in the correction equation. We follow Suyu et al. (2006) to obtain the source-intensity distribution on a grid of pixels given the current iteration's lens potential model. In this source reconstruction approach, the data (observed image) are described by the vector $d_{j}$, where $j=1, \ldots, N_{\mathrm{d}}$ and $N_{\mathrm{d}}$ is the number of data pixels. The source intensity is described by the vector $s_{i}$, where $i=1, \ldots, N_{\mathrm{s}}$ and $N_{\mathrm{s}}$ is the number of source-intensity pixels. The observed image is related to the source intensity via $d_{j}=\mathrm{f}_{j i} s_{i}+n_{j}$, where $\mathrm{f}_{j i}$ is the so-called blurred lensing operator (mapping matrix) that incorporates the lens potential (which governs the deflection of light rays) and the PSF (blurring), ${ }^{10}$ and $n_{j}$ is the noise in the data characterized by the covariance matrix $\mathbf{C}_{\mathrm{D}}$. In the inference of $s_{i}$, we impose a prior on $s_{i}$, which can be thought of as "regularizing" the parameters $s_{i}$ to avoid overfitting to the noise in the data. Following Suyu et al. (2006), we use quadratic forms of the regularization (specifically, zeroth-order, gradient, and curvature forms of regularization). The Bayesian inference of the source-intensity distribution $\left(s_{i}\right)$ given the observed image $\left(d_{j}\right)$ is a linear inversion and is a solved problem. Having

\footnotetext{
$9 \psi$ includes the distance ratio.

${ }^{10}$ Dust extinction, if present, is also included in this mapping matrix $\mathrm{f}_{j i}$.
}

obtained the source intensity, we can calculate the intensity deficit and source-intensity gradients.

We pixelize the lens potential to allow for a flexible parametrization scheme. To solve Equation (3), we cast it into a matrix equation and invert the linear system. To write Equation (3) in a matrix form, we discretize the lens potential on a rectangular grid of $N_{\mathrm{p}}$ pixels (which is less than the number of data pixels $N_{\mathrm{d}}$ so that the potential and source-intensity pixels are not underconstrained) and denote the potential perturbation by $\delta \psi_{i}$, where $i=1, \ldots, N_{\mathrm{p}}$. The intensity deficit on the image grid is $\delta I_{j}=d_{j}-\mathrm{f}_{j i} s_{i}$, where $j=1, \ldots, N_{\mathrm{d}}$ (using the notation from source-intensity reconstruction, $\boldsymbol{d}, \mathbf{f}$, and $\boldsymbol{s}$ are the data vector, the blurred lensing operator, and the source-intensity vector, respectively). Equation (3) now becomes

$$
\delta I=\mathbf{t} \delta \psi+n,
$$

where $\mathbf{t}$ is a $N_{\mathrm{d}} \times N_{\mathrm{p}}$ matrix, which incorporates the PSF, the source-intensity gradient, and the gradient operator that acts on $\delta \boldsymbol{\psi}$ (see the appendix for the explicit form of $\mathbf{t}$ ), and $\boldsymbol{n}$ is the noise in the data. The above equation is equivalent to

$$
d=\mathbf{f} s+\mathbf{t} \delta \psi+n .
$$

We can infer the potential corrections $\delta \boldsymbol{\psi}$ given the data $\boldsymbol{d}$, source intensity $\boldsymbol{s}$, and source-intensity gradients that are encoded in $\mathbf{t}$. In the inference, we impose a prior on $\delta \psi$. The posterior probability distribution is

$$
\overbrace{P\left(\boldsymbol{\delta} \boldsymbol{\psi} \mid \boldsymbol{d}, \mathbf{f}, \boldsymbol{s}, \mathbf{t}, \mu, \mathbf{g}_{\delta \psi}\right)}^{\text {posterior }}=\frac{\overbrace{P(\boldsymbol{d} \mid \boldsymbol{\delta} \boldsymbol{\psi}, \mathbf{t}, \mathbf{f}, \boldsymbol{s})}^{\text {likelihood }} \overbrace{P\left(\boldsymbol{\delta} \boldsymbol{\psi} \mid \mu, \mathbf{g}_{\delta \psi}\right)}^{\text {prior }}}{\underbrace{P\left(\boldsymbol{d} \mid \mathbf{f}, \boldsymbol{s}, \mathbf{t}, \mu, \mathbf{g}_{\delta \psi}\right)}_{\text {evidence }}},
$$

where $\mu$ and $\mathbf{g}_{\delta \psi}$ are the (fixed) strength and form of regularization for the potential correction inversion, and all irrelevant (in)dependences have been dropped. Modeling the noise as Gaussian, the likelihood is

$$
P(\boldsymbol{d} \mid \boldsymbol{\delta} \boldsymbol{\psi}, \mathbf{t}, \mathbf{f}, \boldsymbol{s})=\frac{\exp \left(-E_{\mathrm{D}}(\boldsymbol{d} \mid \boldsymbol{\delta} \boldsymbol{\psi}, \mathbf{t}, \mathbf{f}, \boldsymbol{s})\right)}{Z_{\mathrm{D}}},
$$

where

$$
\begin{aligned}
E_{\mathrm{D}}(\boldsymbol{d} \mid \boldsymbol{\delta} \boldsymbol{\psi}, \mathbf{t}, \mathbf{f}, \boldsymbol{s}) & =\frac{1}{2}(\boldsymbol{d}-\mathbf{f} s-\mathbf{t} \boldsymbol{\delta} \boldsymbol{\psi})^{\mathrm{T}} \mathbf{C}_{\mathrm{D}}^{-1}(\boldsymbol{d}-\mathbf{f} s-\mathbf{t} \boldsymbol{\delta} \boldsymbol{\psi}) \\
& =\frac{1}{2} \chi^{2}
\end{aligned}
$$

and $Z_{\mathrm{D}}$ is the normalization for the probability. We express the prior in the following form:

$$
P\left(\boldsymbol{\delta} \boldsymbol{\psi} \mid \mu, \mathbf{g}_{\delta \psi}\right)=\frac{\exp \left(-\mu E_{\delta \psi}\left(\boldsymbol{\delta} \boldsymbol{\psi} \mid \mathbf{g}_{\delta \psi}\right)\right)}{Z_{\delta \psi}(\mu)} .
$$

We use quadratic forms of the regularizing function $E_{\delta \psi}$. In particular, we use the curvature form of regularization (see for example, Appendix A of Suyu et al. 2006 for an explicit expression of the curvature form of regularization). We use this regularization instead of the zeroth-order or gradient forms because the lens potential should in general be smooth, being the integral of the surface mass density. Curvature regularization 
in the potential corrections effectively corresponds to zerothorder regularization in the surface mass density corrections. This implies a prior preference toward zero surface mass density corrections, thus suppressing the addition of mass to the initial mass model unless the data require it.

Maximizing the posterior of parameters $\delta \boldsymbol{\psi}$, we obtain the most probable solution

$$
\delta \psi_{\mathrm{MP}}=\mathbf{A}^{-1} \boldsymbol{D},
$$

where

$$
\begin{aligned}
\mathbf{A} & =\mathbf{B}+\mu \mathbf{C}, \\
\mathbf{B} & \equiv \nabla \nabla E_{\mathrm{D}}(\boldsymbol{\delta} \boldsymbol{\psi})=\mathbf{t}^{\mathrm{T}} \mathbf{C}_{\mathrm{D}}^{-1} \mathbf{t}, \\
\mathbf{C} & \equiv \nabla \nabla E_{\delta \psi}(\boldsymbol{\delta} \boldsymbol{\psi}), \\
D & =\mathbf{t}^{\mathrm{T}} \mathbf{C}_{\mathrm{D}}^{-1}(\boldsymbol{d}-\mathbf{f} \boldsymbol{s}), \\
\text { and } \nabla & \equiv \frac{\partial}{\partial \boldsymbol{\delta} \boldsymbol{\psi}} .
\end{aligned}
$$

The matrices A, B, and C have dimensions $N_{\mathrm{p}} \times N_{\mathrm{p}}$ and are, by definition, the Hessians of the exponential arguments in the posterior, the likelihood, and the prior probability distributions, respectively.

As discussed in detail in, for example, MacKay (1992) and Suyu et al. (2006), the evidence is irrelevant in the first level of inference where we maximize the posterior of parameters $\delta \psi$ to obtain the most probable parameters $\delta \psi_{\mathrm{MP}}$. However, the evidence is crucial for the second level of inference for model comparison, where a model incorporates the lens potential, PSF, and regularizations of both the source intensity and the potential correction. If we assert that models are equally probable a priori, then the evidence gives the relative probability of the model given the data. In other words, the ratio in the evidence values of two models tells us how much more probable the first model is relative to the second model if we assume that the two models are a priori equally probable. Since the evidence gives only the relative probability, the data set needs to be kept the same for model comparison.

The posterior $\left(P\left(\boldsymbol{\delta} \boldsymbol{\psi} \mid \boldsymbol{d}, \mathbf{f}, \boldsymbol{s}, \mathbf{t}, \mu, \mathbf{g}_{\delta \psi}\right)\right)$ and the evidence $\left(P\left(\boldsymbol{d} \mid \mathbf{f}, \boldsymbol{s}, \mathbf{t}, \mu, \mathbf{g}_{\delta \psi}\right)\right)$ in Equation (6) are conditional on the source-intensity distribution. Ideally, we would have an expression of the posterior for both $s$ and $\delta \boldsymbol{~ : ~}$ $P\left(\boldsymbol{s}, \boldsymbol{\delta} \boldsymbol{\psi} \mid \boldsymbol{d}, \mathbf{f}, \lambda, \mathbf{g}_{\mathrm{s}}, \mathbf{t}, \mu, \mathbf{g}_{\delta \psi}\right)$, where $\lambda$ and $\mathbf{g}_{\mathrm{s}}$ are, respectively, the strength and form of regularization for $s$. We would also obtain the evidence by marginalizing both the source-intensity and the potential correction values, $P\left(\boldsymbol{d} \mid \mathbf{f}, \lambda, \mathbf{g}_{\mathrm{s}}, \mathbf{t}, \mu, \mathbf{g}_{\delta \psi}\right)=$ $\int \mathrm{d} \boldsymbol{s} \mathrm{d} \boldsymbol{\psi} \boldsymbol{P} \mathrm{P}(\boldsymbol{d} \mid \boldsymbol{s}, \delta \psi, \mathbf{f}, \mathbf{t}) \mathrm{P}\left(\boldsymbol{s}, \boldsymbol{\delta} \boldsymbol{\psi} \mid \lambda, \mathbf{g}_{\mathrm{s}}, \mu, \mathbf{g}_{\delta \psi}\right)$. However, due to the iterative nature of the method (i.e., $s$ and $\delta \psi$ are not inferred simultaneously), we do not have such expressions for the posterior and the evidence. Pragmatically, we use the evidence from the source reconstruction (given the corrections $\delta \boldsymbol{\psi}), P\left(\boldsymbol{d} \mid \mathbf{f}, \delta \boldsymbol{\psi}, \lambda, \mathbf{g}_{\mathrm{S}}\right)$, for comparing the potential models, PSF, and regularizations. Specifically, after iterating through the source-intensity reconstructions and lens potential corrections, we use the final corrected lens potential for one last source-intensity reconstruction and use the evidence from this final source reconstruction for comparing models. This approximation is valid provided that the probability distributions of $\delta \psi$ and the regularization constant are sharply peaked at the most probable values. Suyu et al. (2006) showed that the delta function approximation for the regularization constant is acceptable; simulations of the iterative potential reconstruction method suggest that the probability of $\delta \psi$ after the final iteration is sharply peaked. Therefore, the probability of a given potential model, PSF, and form of regularization is $P\left(\mathbf{f}, \mathbf{g}_{\mathrm{S}} \mid \boldsymbol{d}\right) \propto \int \mathrm{d} \lambda \mathrm{d} \boldsymbol{\delta} \boldsymbol{\psi} \mathrm{P}\left(\boldsymbol{d} \mid \mathbf{f}, \boldsymbol{\delta} \boldsymbol{\psi}, \lambda, \mathbf{g}_{\mathrm{S}}\right) \mathrm{P}\left(\mathbf{f}, \mathbf{g}_{\mathrm{S}}\right) \sim$ $\mathrm{P}\left(\boldsymbol{d} \mid \mathbf{f}, \hat{\boldsymbol{\delta}} \hat{\boldsymbol{\psi}}, \hat{\lambda}, \mathbf{g}_{\mathrm{S}}\right) \mathrm{P}\left(\mathbf{f}, \mathbf{g}_{\mathrm{S}}\right)$, where $\hat{\boldsymbol{\delta}} \hat{\psi}$ and $\hat{\lambda}$ are the most probable solutions. Assuming that all models are equally probable a priori (i.e., $P\left(\mathbf{f}, \mathbf{g}_{\mathrm{S}}\right)$ is constant), the evidence from the source reconstruction serves as a reasonable proxy to use for model comparison.

There is an uncertainty associated with the evidence values due to finite source-intensity resolution as a result of the source pixelization. The source reconstruction region is initially chosen such that the mapped source region on the image plane encloses the Einstein ring. This ensures that the source region contains the entire source-intensity distribution. Throughout the iterative pixelated potential reconstruction, the source region and pixelization are kept the same. In the final source reconstruction for evidence computation, the evidence value depends on the pixelated source region because the goodness of fit on the image plane generally changes, especially in areas of significant intensity gradients, as one shifts the source region. To estimate the uncertainty in the evidence values, we perform the last source reconstruction for various source regions that are shifted by a fraction of a pixel from the optimized one in the potential reconstruction. The range of the resulting evidence values for the various source regions then allow us to quantify the uncertainty in the evidence. In addition to the uncertainty due to source pixelization, the evidence also depends on the amount of regularization on $\delta \boldsymbol{\psi}$, which is discussed in Section 2.2.2.

\subsubsection{Technicalities of the Pixelated Potential Reconstruction}

Solving for the potential perturbations is very similar to solving for the source-intensity distribution in Suyu et al. (2006) except for the following technical details.

1. In each iteration, the perturbative potential correction is obtained only in an annular region instead of over the entire lens potential grid due to the need for the source-intensity gradient (see Equation (3)) to be measurable. Since the extended source intensity is only non-negligible near the Einstein ring, we only have information about the sourceintensity gradients in this region. In practice, the annular region is the mapping of the finite source reconstruction grid that encloses the extended source with a minimal number of source pixels (for computational efficiency). The annulus of potential corrections obtained at each iteration is extrapolated for the next iteration by minimizing the curvature in the potential corrections. This allows the shape of the annular region to change as needed when the lens potential gets corrected. In addition, the forms of the regularization matrix, as discussed in Appendix A of Suyu et al. (2006), are modified accordingly to take into account the nonrectangular reconstruction region (described in more detail in the third point below).

2. Since Equation (5) is a perturbative equation in $\delta \boldsymbol{\psi}$, the inversion needs to be over-regularized to enforce a small correction in each iteration. Empirically, we set the regularization constant, $\mu$, at roughly the peak of the function $\mu E_{\delta \psi}$ (within a factor of 10 ), which corresponds to the value before which the prior dominates. The resulting evidence value from the final source-intensity reconstruction weakly depends on the value of $\mu$, and we include this dependence in the uncertainty of the evidence value. 
3 . The potential corrections are generally nonzero at the edge of the annular reconstruction region. This calls for slightly different structures of regularization compared to those written in Appendix A in Suyu et al. (2006) for source-intensity reconstruction (since the source grids are chosen to enclose the entire extended source such that edge pixels have nearly zero intensities). The regularizations are still based on derivatives of $\delta \boldsymbol{\psi}$; however, no patching with lower derivatives should be used for the edge pixels because the zeroth-order regularization at the top/right edge will incorrectly enforce the $\delta \psi$ values to zero in those areas. The absence of the lower derivative patches leads to a singular regularization matrix, ${ }^{11}$ which is problematic for evaluating the Bayesian evidence for lens potential correction. However, since we do not use the evidence values to compare the forms of regularization for the potential corrections (because we use only the curvature form) nor to compare the lens potential and PSF model, the revised structure of regularization is acceptable. We have found this structure of regularization for potential corrections to work for various types of sources (with varying sizes, shapes, number of components, etc.).

In the source reconstruction steps of this iterative scheme, we discover by using simulated data that over-regularizing the source reconstruction in early iterations helps the process to converge. This is because initial guess potentials that are significantly perturbed from the true potential often lead to highly discontinuous source distributions when optimally regularized (corresponding to maximal Bayesian evidence, which balances the goodness of fit and the prior), and over-regularization would give a more regularized source-intensity gradient for the potential correction. Unfortunately, we do not have an objective way of setting this over-regularization factor for the source reconstruction. Currently, at each source reconstruction iteration, we set the over-regularization factor such that the magnitude of the intensity deficit is at approximately the same level as that from the optimally-regularized case but with a smoother sourceintensity distribution for numerical derivatives. This scheme ensures that we do not over-regularize when we are close to the true potential. Based on simulated test runs, the recovery of the true potential depends on the amount of over-regularization. When the initial guess is far from the true potential, over-regularization in the early iterations is crucial for convergence. We find that it is better to over-regularize in excess than in deficit. Too much over-regularization simply leads to more iterations to converge, whereas too little over-regularization may not converge at all.

For each iteration of source-intensity reconstruction, there is also a mask on the source plane to exclude source pixels that either (1) are not mapped by that iteration's lens potential on the data grid or (2) have no neighboring pixels for the computation of numerical derivatives. We generalize the regularizing function for this nonrectangular reconstruction region to have the right-most and top-most pixels (pixels adjacent to the edge or adjacent to the masked source pixels) patched with lower derivatives as we did for the edge pixels in Appendix A of Suyu et al. (2006). This patching ensures that the regularization matrix is nonsingular for the evaluation of the Bayesian evidence.

Based on simulated test runs, we find that a practical stopping criterion for the iterative procedure is to terminate when the

\footnotetext{
$\overline{11}$ Having a singular regularization matrix $(\mathbf{C})$ does not prevent one from calculating $\delta \boldsymbol{\psi}$ because the matrix for inversion $(\mathbf{A}=\mathbf{B}+\mu \mathbf{C})$ is, in general, nonsingular.
}

relative potential corrections between all image pairs are $\left(\delta \psi_{1}-\right.$ $\left.\delta \psi_{2}\right) /\left(\psi_{1}-\psi_{2}\right)<0.1 \%$, where 1 and 2 label the images in any pair. After this criterion is reached, further iterations give a negligible contribution to the predicted Fermat potential differences between the images.

\subsubsection{Mass-Sheet Degeneracy}

The restriction to using only isophotal data implies that the potential correction we obtain at each iteration may be affected by the "mass-sheet degeneracy" (Falco et al. 1985). However, the addition of mass sheets is suppressed by the curvature form of the regularization for the potential correction and also by the large amount of over-regularization. We refer to Kochanek et al. (2006) and Paper II for a detailed description of the mass-sheet degeneracy; here we review a few key points that are relevant for the potential corrections. In essence, an arbitrary symmetric paraboloid, gradient sheet, and constant can be added to the potential without changing the predicted lensed image:

$$
\psi_{\nu}(\vec{\theta})=\frac{1-v}{2}|\vec{\theta}|^{2}+\vec{a} \cdot \vec{\theta}+c+v \psi(\vec{\theta})
$$

where $\psi(\vec{\theta})$ is the original potential, $\psi_{v}(\vec{\theta})$ is the transformed potential, and $v, \vec{a}$, and $c$ are constants. The constants $\vec{a}$ and $c$ have no physical effects on the lens systems as they merely change the origin on the source plane (which is unknowable) and change the zero point of the potential (which is not observable). The parameter $1-v$ refers to the amount of mass sheet, which can be seen in the corresponding convergence transformation: $\kappa_{\nu}(\vec{\theta})=(1-v)+\nu \kappa(\vec{\theta})$. To make sure we remain "close" to the initial potential model, we set $v=1$ and fix three points in the corrected potential after each iteration to the corresponding values of the initial potential. Setting $v=1$ ensures that the size of the extended source intensity remains approximately the same, and the three fixed points allow us to solve for $\vec{a}$ and $c$ in Equation (12) to remove irrelevant gradient sheets and constants in the reconstructed potential. We choose the three points to be three of the four (top, left, right, and bottom) locations of the annular reconstruction region that are midway in thickness between the annular edges. The three points are usually chosen to be at places with lower surface brightness in the ring. This technique of "fixing" the mass-sheet degeneracy is demonstrated in Section 2.2.4 using simulated data.

\subsubsection{Summary}

To summarize, the steps for the iterative and perturbative potential reconstruction scheme via matrices are as follows. (1) Reconstruct the source-intensity distribution given the initial (or corrected) lens potential based on Suyu et al. (2006). (2) Compute the intensity deficit and the source-intensity gradient. (3) Solve Equation (5) for the potential corrections $\delta \psi$ in the annulus of reconstruction. (4) Update the current potential using Equation (2): $\psi_{\text {next iteration }}=\psi_{\text {current iteration }}-\delta \psi$. (5) Transform the corrected potential $\psi_{\text {next iteration via Equation (12) so that }}$ $v=1$ and the transformed corrected potential has the same values as the initial potential at the three fixed points. (6) Extrapolate the transformed corrected potential for the next iteration. (7) Interpolate the transformed corrected potential onto the resolution of the data grid for the next iteration's source reconstruction. (8) Repeat the process using the extrapolated and finely gridded reconstructed potential, and stop the process when the relative potential correction between any pair of images is $<0.1 \%$. 

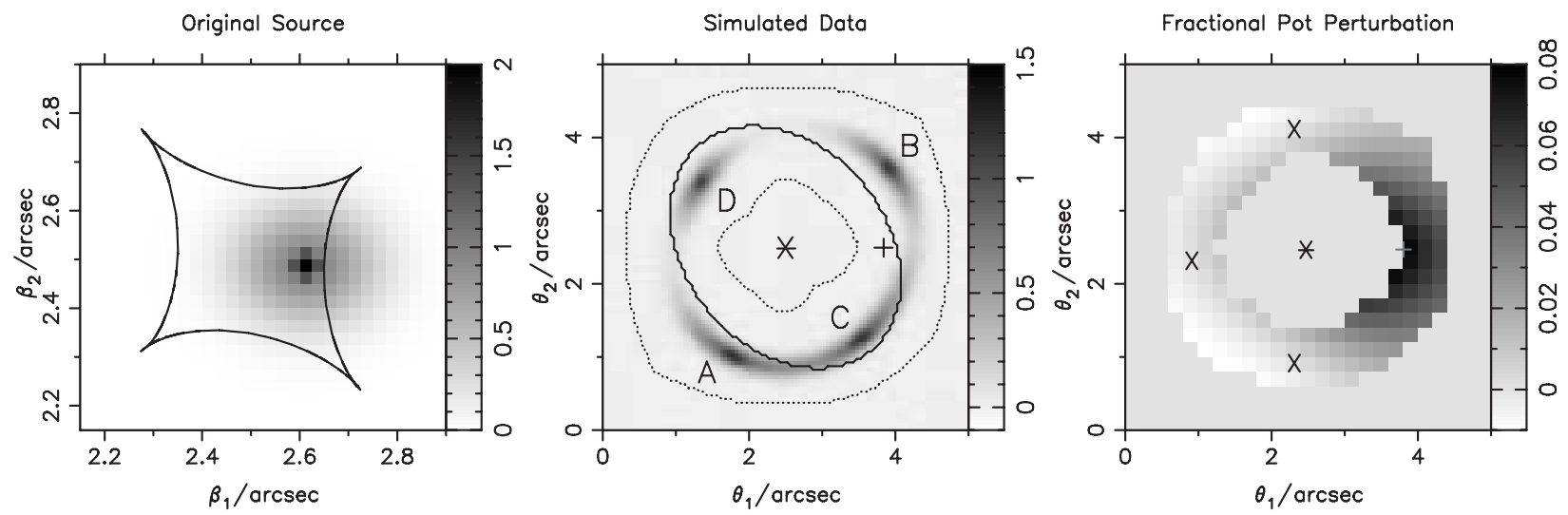

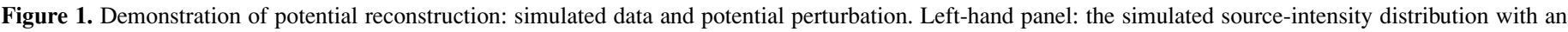

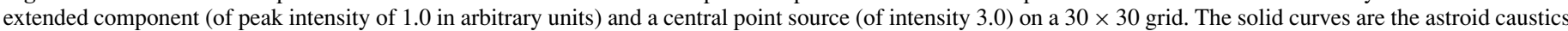

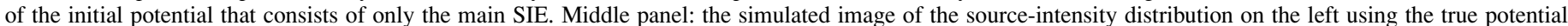

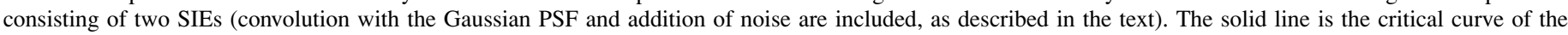

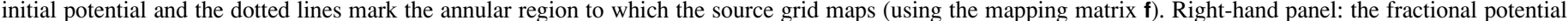

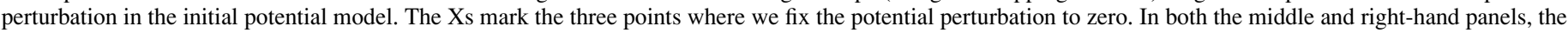
asterisk and the plus sign indicate the positions of the main SIE component and the perturbing SIE component, respectively.

Table 1

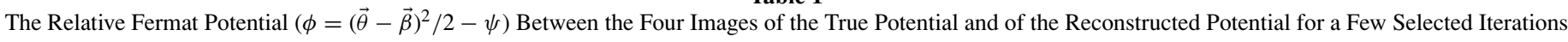

\begin{tabular}{lcccc}
\hline \hline Potential & $\phi_{A B}$ & $\phi_{C B}$ & $\phi_{D B}$ & $\begin{array}{c}\text { Source Position } \\
(\operatorname{arcsec})\end{array}$ \\
\hline True & 0.141 & 0.234 & 0.437 & \\
Initial & $0.172 \pm 0.189$ & $0.228 \pm 0.156$ & $0.437 \pm 0.041$ & $(2.587,2.483) \pm(0.013,0.076)$ \\
Iteration $=0$ & $0.178 \pm 0.070$ & $0.246 \pm 0.068$ & $0.479 \pm 0.010$ & $(2.608,2.483) \pm(0.006,0.034)$ \\
Iteration $=2$ & $0.161 \pm 0.011$ & $0.242 \pm 0.010$ & $0.471 \pm 0.011$ & $(2.623,2.484) \pm(0.005,0.005)$ \\
Iteration $=9$ & $0.151 \pm 0.006$ & $0.244 \pm 0.004$ & $0.454 \pm 0.006$ & $(2.621,2.484) \pm(0.003,0.002)$ \\
\hline$v$ & 0.96 & & &
\end{tabular}

Notes. We use the average source position of the four source positions for the computation of the Fermat potential. The four source positions deviate by $\sim 0^{\prime \prime} .1$ in the initial model, and agree within $\sim 0.005$ at iteration $=9$. The uncertainties in the predicted relative Fermat potential are due to the uncertainties in the source position. The good agreement between the predicted Fermat potential values for the initial potential and the true values is coincidental due to the use of the average source position.

\section{DEMONSTRATION: POTENTIAL PERTURBATION DUE TO AN INVISIBLE MASS CLUMP}

In the previous section, we have outlined a method of pixelated potential reconstruction. In this section, we will demonstrate this method using simulated data with a lens consisting of two mass components.

\subsection{Simulated Data}

We use singular isothermal ellipsoid (SIE) potentials (Kormann et al. 1994) to test the potential reconstruction method. For this demonstration, we let the lens be comprised of two SIEs at the same redshift $z_{\mathrm{d}}=0.3$ : a main component and a perturber. The main lens has a one-dimensional velocity dispersion of $260 \mathrm{~km} \mathrm{~s}^{-1}$, an axis ratio of 0.75 , and a semimajor axis position angle of $45^{\circ}$ (from vertical in the counterclockwise direction). The (arbitrary) origin of the coordinates is set such that the lens is centered at $\left(2^{\prime \prime} .5,2^{\prime \prime} .5\right)$, the center of the $5^{\prime \prime} \times 5^{\prime \prime}$ image. The perturbing SIE is centered at $\left(3^{\prime \prime} .8,2\right.$. .5$)$ with a velocity dispersion of $50 \mathrm{~km} \mathrm{~s}^{-1}$, axis ratio of 0.60 , and semimajor axis position angle of $70^{\circ}$. The exact potential is the sum of these two SIEs. We model the source intensity as an elliptical distribution inside the caustics at $z_{\mathrm{s}}=3.0$ with an extended component (of peak intensity of 1.0 in arbitrary units) and a central point source (of intensity 3.0). This source is chosen such that the lensed im- age resembles B1608+656. We use $100 \times 100$ image pixels each of size 0'.05 (typical pixel size of the HST Advanced Camera for Surveys (ACS)), $30 \times 30$ source pixels each of size 0 '.025, and $25 \times 25$ potential pixels each of size $00^{\prime} 2$. To obtain the simulated data, we map the source-intensity distribution to the image plane using the exact lens potential and the lens equation, convolve the lensed image with a Gaussian PSF whose FWHM $=00^{\prime \prime} 15$, and add Gaussian noise of variance 0.015 . Figure 1 shows the simulated source in the left-hand panel and the simulated noisy data image in the middle panel. The Fermat potential difference between the images are listed in Table 1. The images are labeled by $\mathrm{A}, \mathrm{B}, \mathrm{C}, \mathrm{D}$, and their locations are (1".77, 1".02), (3".90, 3".59), $\left(3^{\prime \prime} .54,1^{\prime \prime} .26\right)$, and $\left(1^{\prime \prime} .34,3^{\prime \prime} .38\right)$, respectively.

\subsection{Iterative and Perturbative Potential Corrections}

We take the initial guess of the lens potential to be the main SIE component but with the position angle changed from $45^{\circ}$ to $40^{\circ}$. This corresponds to a typical scenario where the perturbing SIE is faint/dark so that it is not detected in the image, and hence is not incorporated in the smooth parametrized model of the main SIE component. The rotation in the position angle of the main SIE component corresponds to a situation where the mass of the galaxy does not strictly follow the light, but the position angle of the lens mass distribution is 
initially adopted from the position angle of the lens galaxy light. Here and after, "initial potential" refers to this initial guess of the potential model (as opposed to the true/exact potential). Figure 1 shows the potential perturbation relative to the initial potential in the right-hand panel. In obtaining this plot, the initial potential has a constant gradient plane and offset added such that the top, left, and bottom midpoints in the annulus (marked by Xs in the plot) are fixed to the true potential with zero potential perturbation (as described in the passage following Equation (12)). In the iterative potential reconstruction process, the reconstructed potential at each iteration also has these three points in the annulus fixed to the initial model. The locations of the three fixed points have no impact on recovering the true potential when the source is extended enough to form an Einstein ring on the image plane. However, if the source is compact, then locations of the three points do matter and they are chosen to be at places where the information content (image intensity) is low.

We perform 10 iterations of the perturbative potential correction method outlined in Section 2.2. The iterations are labeled "PI" from 0 to 9. For each source reconstruction iteration, we adopt the curvature form of regularization and use the sourceintensity reconstruction for the evaluation of the source-intensity gradients that are needed for the potential correction. The source inversions are over-regularized in early iterations in order to obtain smooth source reconstructions for evaluating the gradients. For each potential correction iteration, we use the curvature form of regularization and set the regularization constant for the potential reconstruction to be $10 \times$ the value of $\mu$ where $\mu E_{\delta \psi}$ peaks in iteration $=0$. This regularization value is $\sim 10^{8}$ and is used for all subsequent iterations (since we find that the peak in $\mu E_{\delta \psi}$ changes little as the iterations proceed). For comparison, the "optimal" regularization constant is $\sim 10^{2}$ at iteration $=0$ and is $\sim 10^{7}$ at iteration $=9$. Therefore, the potential reconstruction inversions are heavily over-regularized in the early iterations to keep the corrections to first order; as the lens potential gets corrected, the amount of over-regularization diminishes as the inversion approaches the linear regime with small intensity deficits. We show figures of source reconstructions and potential corrections for some, but not all, of the iterations.

The top row of Figure 2 shows the results of PI $=0$. The over-regularized reconstructed source in the left-hand panel does not resemble the original source, and the (normalized) image residual in the middle-left panel shows prominent arc features due to the presence of both the misaligned initial model and the SIE potential perturbation. The reconstructed $\delta \boldsymbol{\psi}$ in the middle-right panel is of the same structures as the exact $\delta \psi$ in Figure 1, though the magnitude is smaller due to the correction being a perturbative one. A plot of the image residual after correction $(=\delta \boldsymbol{I}-\mathbf{t} \delta \boldsymbol{\psi})$ continues to show arc features though less prominent than in the top middle-left panel in Figure 2. The same image residual plot with the true potential perturbation also shows similar arc features, which indicates that Equation (3) is indeed a perturbative equation and thus justifies the overregularization in the potential correction step.

The second row of Figure 2 shows the results of PI $=2$. The reconstructed source in the left-hand panel better resembles the original source in Figure 1. The amount of misfit in the image residual has decreased in the middle-left panel, signaling that we are correcting toward the true potential. The middle-right panel is the potential correction in $\mathrm{PI}=2$, and the right-hand panel is the amount of perturbation that remains after PI $=2$. The amount of potential perturbation remaining is closer to zero compared to the top row, which is a sign that the iterative method converges.

The bottom panels in Figure 2 show the results of PI $=$ 9 , the last iteration. The source is faithfully recovered in the left-hand panel, resulting in negligible image residual in the middle-left panel (reduced $\chi^{2}=1.02$ inside the annulus ${ }^{12}$ ). The centroid of the source is slightly shifted compared to the original because of our adding constant gradients to fix the three points in the potential corrections. The absolute position of the source is irrelevant as we can arbitrarily set the coordinates; it is only the relative positions on the source plane that matter. The source positions are shifted relative to the plotted caustic curve only because these caustic curves are the ones from the initial potential guess (they were not computed for the reconstructed potential due to the low resolution in the reconstructed potential grids). If we were to plot the caustic curve of the corrected potential, we would find no overall shift in the source with respect to the caustic curve. The middle right panel shows the final iteration's potential correction, which is barely visible due to the negligible image residual left to correct. The righthand panel shows that most of the potential perturbation to the true potential has been corrected, though there is still some left. However, this amount of remaining uncorrected potential perturbation leads to image residuals that are effectively masked by the noise in the data. We have thus reached the limit in the potential correction that is set by noise in the data.

Table 1 lists the predicted Fermat potential differences for the initial potential guess and for the corrected potential in PI $=0,2$, and 9. We use the average source position (also listed in the table) of the four mapped source positions for the computation of the Fermat potential. The uncertainty in the predicted Fermat potential difference comes from the error in the source position due to discrepancies in the mapped source positions of the four images. The mapped source positions agree within $\sim 0$ 0'005 (i.e., within a fifth of a source pixel) in the final iteration, a significant improvement to $\sim 00^{\prime} 1$ in the initial potential. The convergent Fermat potential differences in PI = 9 are systematically higher than the true Fermat potential differences. This is because lensing only allows us to recover the Fermat potential differences up to a constant factor due to the mass-sheet degeneracy. The transformation in Equation (12) would scale the Fermat potential difference by a factor of $v$. The last row in Table 1 shows that a mass-sheet transformation with $v=0.96$ leads to the predicted Fermat potential values agreeing with the true values within the uncertainties. We expect this particular simulation's reconstructed pixelated potential to be different from the true potential by a mass-sheet transformation of $v \sim 0.96$ due to the unaccounted mass of the secondary SIE ( $\sim 4 \%$ of the primary SIE) in the initial model. In the iterative potential corrections, mass additions are suppressed in the annulus due to the regularization. This breaks the masssheet degeneracy, but underestimates the total mass within the annulus (the SIE perturber was not included in the initial model): the reconstructed potential, therefore, continues to have a deficit of mass in the annulus. Since the value of the convergence in the annulus is generally less than 1 , the reconstructed potential is thus approximately a mass-sheet transformation of the true potential with the mass deficit in the form of a constant sheet.

\footnotetext{
12 The reduced $\chi^{2}$ is given by $\chi^{2} /\left(N_{\text {pix in annulus }}-\gamma\right)$, where $N_{\text {pix in annulus }}$ is the number of data pixels in the annulus that encloses the ring and $\gamma$ is an estimate of the number of "effective" parameters (e.g., MacKay 1992; Suyu et al. 2006).
} 
Reconstructed Source: it $=0$

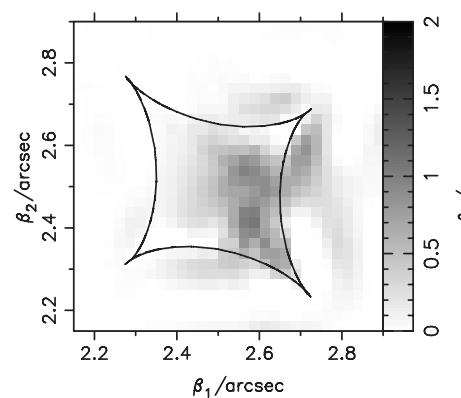

Reconstructed Source: it=2

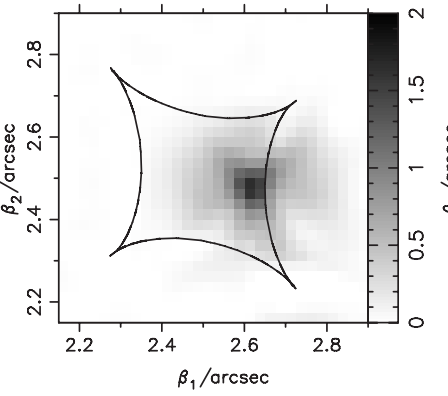

Reconstructed Source: it $=9$

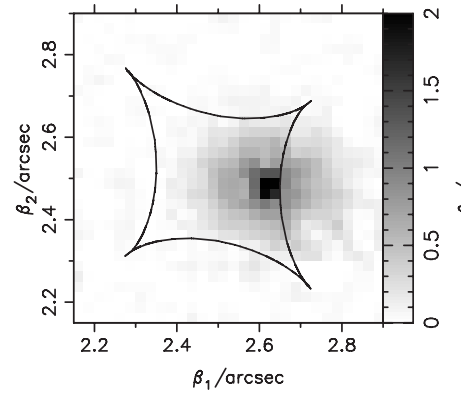

Normalized Image Res: it $=0$

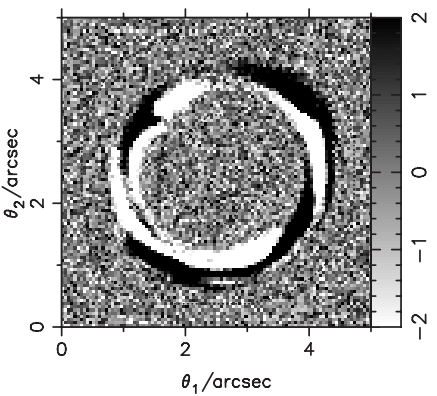

Normalized Image Res: it $=2$

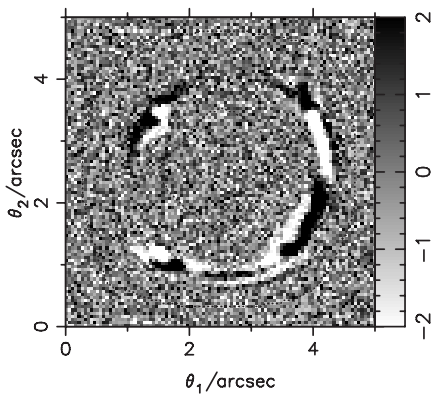

Normalized Image Res: it $=9$

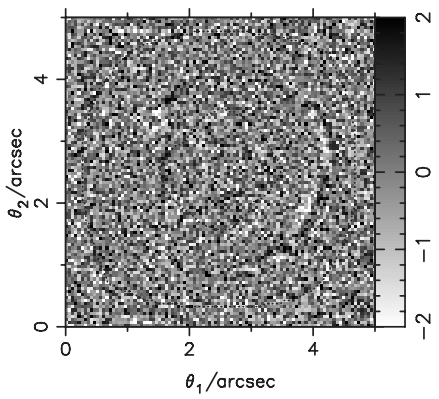

Potential Correction: it $=0$

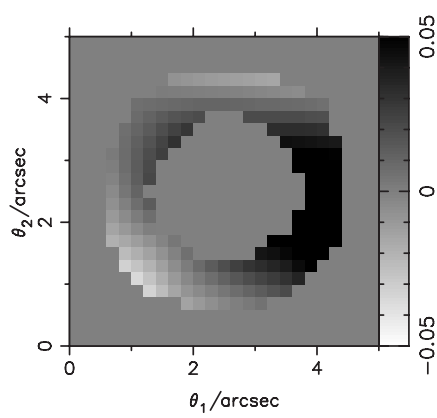

Potential Correction: it $=2$

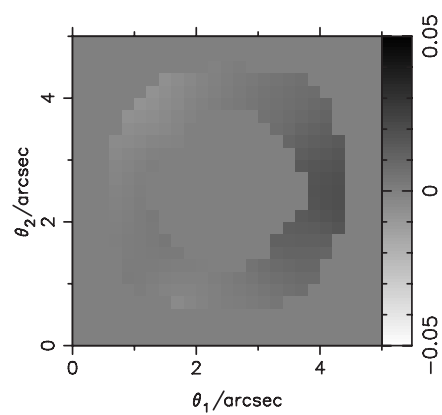

Potential Correction: it $=9$

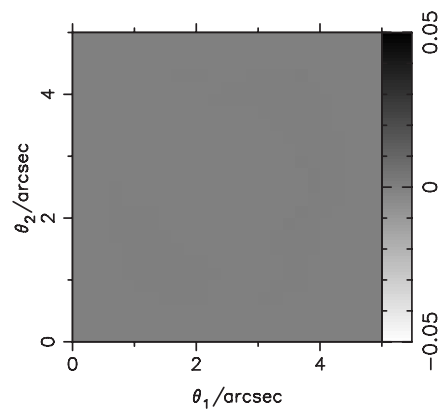

Remaining Pot Pert: it $=0$

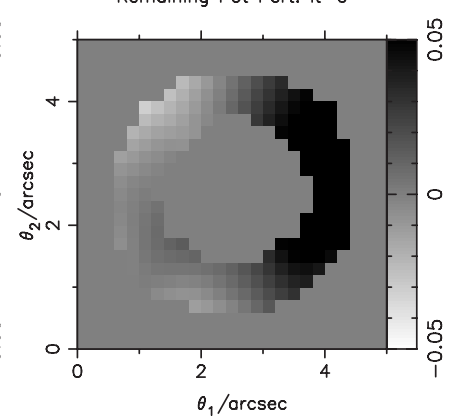

Remaining Pot Pert: it $=2$

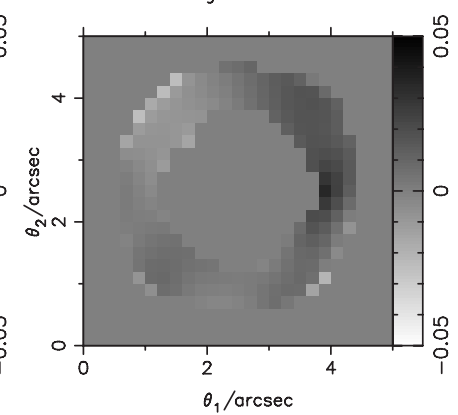

Remaining Pot Pert: it $=9$

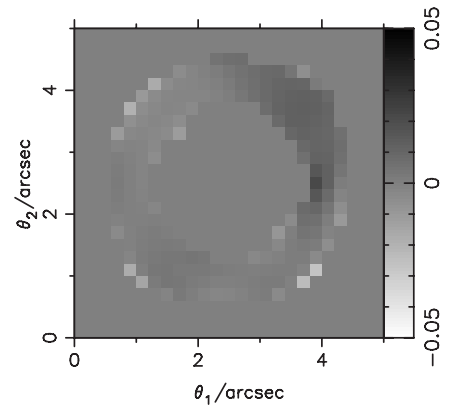

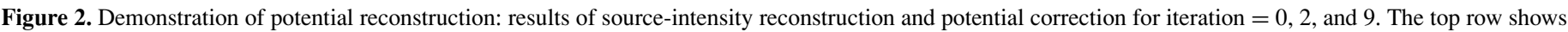

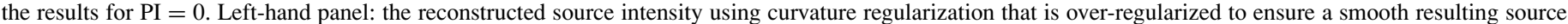

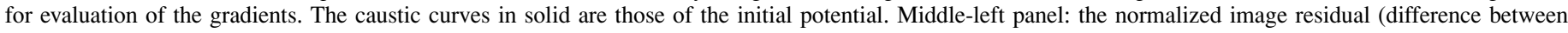

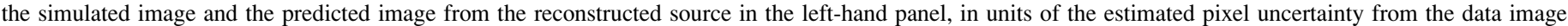

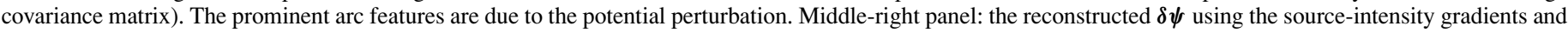

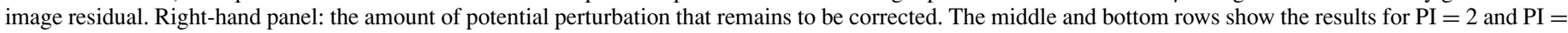

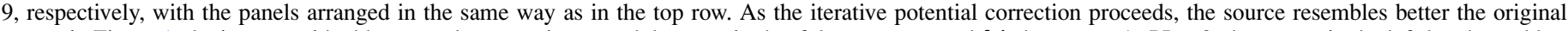

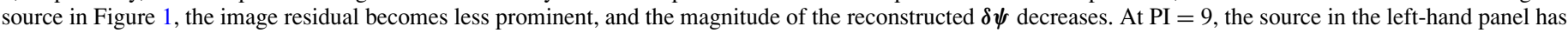

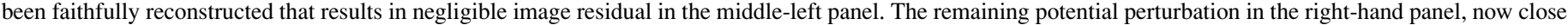
to zero, cannot be fully corrected due to the noise in the data.

The simulation we have shown is one of the worst-case scenarios where even the total mass of the initial lens model enclosed within the Einstein ring is wrong. For initial potential models that have the correct amount of mass within the Einstein ring (this enclosed mass is what lensing can robustly measure to $\sim 1 \%-2 \%$ accuracy in real systems) and with the mass-sheet degeneracy broken (using external information such as stellar dynamics), the reconstructed potential would faithfully recover the Fermat potential.

\subsection{Discussion}

This demonstration shows that the iterative and perturbative potential reconstruction method works in practice. Using simulated data, we find that potential perturbations $\lesssim 5 \%$ (which may correspond to as much as $\sim 20 \%$ in the relative potential perturbations between image pairs) are correctable, though the actual amount depends on the amount of over-regularization for both the source inversion and the potential correction, and on the extendedness of the source-intensity distribution. In the case where the solution converges, the magnitudes of the relative potential corrections between image pairs steadily decrease, and we end the iterative procedure when the stopping criterion (described in Section 2.2) is met.

Regarding the size of the source-intensity distribution, the more extended a source is, the better we can recover the potential. When the source is extended enough to be lensed into a closed ring, the true potential can be fully recovered (up to the limit set by the noise in the data) from potential corrections based on Equation (5). When the source is extended to cover about half of the Einstein ring, then the corrected potential faithfully reproduces the source with negligible image residual, but the relative Fermat potentials may not be recovered due to a slight relative offset in the potential between the images. This is because the "connecting characteristics" (see Suyu \& Blandford 2006) that fix the potential difference between the images go through regions without much signal (light of the lensed source). Therefore, the potential is locally corrected at 
regions near the images (where there is light), but the global offset between the regions cannot be determined.

For sources that are small in extent, the potential correction also depends on the points we choose to fix to the initial potential model. Since an isolated image is generally more prone to having its potential be offset relative to the other images, we set two of the three fixed points in the gaps on both sides of the most isolated image and one point near the connecting images.

We find that a wrong PSF model (e.g., of a different width) would lead to intensity deficit that would not be correctable by the iterative potential reconstruction method. Therefore, an uncorrectable image residual is a sign that our model of the system (other than the lens potential) is wrong.

The potential grid that we used was $25 \times 25$, which we find to be a good balance between the number of degrees of freedom and goodness of fit. The higher the number of potential pixels, the better one can fit to the image residual; however, in this case, it is also more probable to have degenerate solutions. The Bayesian evidence from the source reconstruction in principle can be used to compare the different potential grids. In general, we find that a potential grid that is $\sim 4$ times coarser than the image grid works well.

In Section 4, we generalize this iterative potential reconstruction method, which has been shown to work on simulated data, to treat real gravitational lens images such as B1608+656.

\section{GENERALIZATION TO REALISTIC DATA: INCORPORATING DUST EXTINCTION AND LENS GALAXY LIGHT}

In the previous section, we have demonstrated the method of pixelated potential reconstruction using simulated data. In the mock data, only the image of lensed source was there; in reality, there would also be light from the lens galaxy. Furthermore, in some cases, such as B1608+656, dust is present and absorbs light from both the source galaxy and the lens galaxy. Based on results of the previous section, an accurate extraction of the light from the lensed extended source is crucial for reconstructing the lens potential. Therefore, we will generalize the formalism given in Section 2 to incorporate the lens galaxy light and dust.

Suppose that we have a set of PSF, dust, and lens galaxy light models (the process of obtaining these models is described in detail in Section 5), a lens potential model, and the observed image. Separating the observed image into two components, the lensed source and the lens galaxy, we can model the observed image (as a vector for the intensities of the image pixels) as

$$
\boldsymbol{d}=\overbrace{\mathrm{B} \cdot \mathrm{K} \cdot \mathrm{L} \cdot \boldsymbol{s}}^{\text {lensed extended source }}+\overbrace{\mathrm{B} \cdot \mathrm{K} \cdot \boldsymbol{l}}^{\text {lens galaxy }}+\boldsymbol{n}
$$

where $\mathbf{B}$ is a PSF blurring matrix, $\mathbf{K}$ is a dust extinction matrix, $\mathbf{L}$ is the lensing matrix (containing the lens potential model), $\boldsymbol{s}$ is the source-intensity distribution, $\boldsymbol{l}$ is the lens galaxy intensity distribution, and $\boldsymbol{n}$ is the noise in the data characterized by the covariance matrix $\mathbf{C}_{\mathrm{D}}$. This is an extended version of the equation $\boldsymbol{d}=\mathbf{f} \boldsymbol{s}+\boldsymbol{n}$ in Suyu et al. (2006) with $\mathbf{f}$ replaced by $\mathbf{B} \cdot \mathbf{K} \cdot \mathbf{L}$ and $\boldsymbol{d}$ replaced by $\boldsymbol{d}-\mathbf{B} \cdot \mathbf{K} \cdot \boldsymbol{l}$. The order of the matrix products in both terms are obtained by tracing backwards along the light rays: we first encounter the PSF blurring from the telescope (B), then dust extinction (K) in the lens plane, then the strong lensing effects (L) in the case of the lensed source, and finally the origin of light ( $s$ or $l$ ).

Here we assume that the dust lies in a screen in front of the lensed source and the lens galaxy. This assumption is not strictly valid for the lens galaxy if the dust were to have originated from G2 (Surpi \& Blandford 2003). In this case, the dust and stars are mingled together in the lens galaxy. It is beyond the scope of this paper to treat this mixed light and dust problem. However, we note that the dust screen assumption is acceptable since the aim is to obtain an accurate lensed source-intensity distribution (for which the dust screen assumption is valid) and not the lens galaxy intensity distribution near the core where the mixing effects would dominate. Furthermore, in simple toy models, where either the dust and stars are uniformly mixed or the dust is a screen lying inside the lens galaxy, we find that the extinction of the lens galaxy light is well approximated as extinction by a foreground dust screen with a reduced visual extinction. Our simple foreground dust screen model thus provides an effective extinction that incorporates the reduced extinction for the lens and the full extinction by a foreground dust screen for the lensed source.

If the lensed source contains a bright core such as an active galactic nucleus (AGN), then we could consider extending Equation (13) and model the observed image as

$$
\boldsymbol{d}=\mathbf{B} \cdot \mathbf{K} \cdot \mathbf{L} \cdot \boldsymbol{s}+\sum_{i=1}^{N_{\text {images }}} \mathrm{K}_{i} \alpha_{i} \operatorname{PSF}\left(\vec{\theta}_{i}\right)+\mathbf{B} \cdot \mathbf{K} \cdot \boldsymbol{l}+\boldsymbol{n},
$$

where the light from the extended part of the host (the first term) would be modeled separately from that from the point sources (the second term) and $\alpha_{i}$ are the intensities (flux per unit solid angle in a pixel) of the point sources (which are generally not the same for all images due to finite resolution-both lensing and microlensing give rise to different magnification of the pointlike source - and, in the case of a time-varying core, time delay difference). However, it is the extended image surface brightness that provides the information needed to reconstruct the lens potential. For B1608+656, by taking into account the errors in the modeling associated with the presence of the point sources (see Section 5.2.1), we will find that a separate modeling of the point sources is not necessary for reconstructing the lens potential.

Given $\mathbf{B}, \mathbf{K}, \boldsymbol{l}, \mathbf{L}$, and $\boldsymbol{d}$, one can solve for the most probable source-intensity distribution $s_{\mathrm{MP}}$, as in Suyu et al. (2006). Furthermore, one can use the Bayesian evidence of the source reconstruction to rank different models of PSF, dust extinction, lens galaxy light, and lens potential (see Section 2.2.1). When we compare models, we mark an annular region enclosing the Einstein ring and use the same annulus of data for all models (where models refer collectively to the lens potential, PSF, dust, lens galaxy light, and regularization). For the chosen data set, we determine the source region that maps to the annular region and reconstructs the source intensities in this region. The shape of this source region is generally not rectangular, so we generalize the regularization schemes in Appendix A of Suyu et al. (2006) to patch the right-most and top-most pixels (pixels adjacent to the edge of grid or adjacent to the unmapped source pixels) with lower derivatives. We will use the Bayesian evidence values from the source reconstruction in Sections 5 and 6 to compare various PSF, dust, lens galaxy light and lens potential models for B1608+656.

To include the effects of galaxy light and dust in the pixelated potential reconstruction method, we incorporate $\mathbf{K}$ and $\boldsymbol{l}$ into Equation (5) as in Equation (13), and include $\mathbf{K}$ into $\mathbf{t}$ (see the Appendix for this inclusion). After these adjustments, we can iteratively correct for the lens potential in real systems given a PSF, a dust, and a lens galaxy light model based on the machinery we developed in the previous sections. 
Table 2

HST Observations of B1608+656

\begin{tabular}{|c|c|c|c|c|c|c|}
\hline $\begin{array}{c}\text { Proposal } \\
\text { PI }\end{array}$ & $\begin{array}{c}\text { Proposal } \\
\text { ID }\end{array}$ & Date & Instrument & Filter & Exposures & $\begin{array}{l}\text { Exposure Time } \\
\text { (s) }\end{array}$ \\
\hline \multirow[t]{16}{*}{ C. Fassnacht } & 10158 & 2004 Aug 24 & $\mathrm{ACS} / \mathrm{WFC}$ & F606W & 4 & 609 \\
\hline & & & & & 4 & 646 \\
\hline & & & & F814W & 4 & 632 \\
\hline & & & & & 4 & 646 \\
\hline & & 2004 Aug 25 & ACS/WFC & F606W & 8 & 609 \\
\hline & & & & & 8 & 646 \\
\hline & & & & F814W & 8 & 632 \\
\hline & & & & & 8 & 646 \\
\hline & & 2004 Aug 29 & ACS/WFC & F606W & 4 & 609 \\
\hline & & & & & 4 & 646 \\
\hline & & & & F814W & 4 & 632 \\
\hline & & & & & 4 & 646 \\
\hline & & 2004 Sep 17 & $\mathrm{ACS} / \mathrm{WFC}$ & F606W & 4 & 609 \\
\hline & & & & F814W & 4 & 632 \\
\hline & & & & & 4 & 646 \\
\hline & & & & & 4 & 646 \\
\hline \multirow[t]{3}{*}{ A. Readhead } & 7422 & 1998 Feb 7 & NIC1 & F160W & 5 & 3840 \\
\hline & & & & & 1 & 2048 \\
\hline & & & & & 1 & 896 \\
\hline
\end{tabular}

To conclude, we have outlined and demonstrated an iterative and perturbative potential correction scheme where the accuracy in the reconstruction is limited by the noise in the data. The inputs for this method are an initial guess of the lens potential as well as assumptions regarding the PSF, dust, and lens galaxy light. The outputs are the reconstructed potential on a grid of pixels, the reconstructed source-intensity distribution, and the Bayesian evidence from source reconstruction, given the assumptions. Our goal is to apply this method to the wellobserved lens system B1608+656, and we begin by describing our HST observations of B1608+656 in Section 5.

\section{IMAGE PROCESSING OF B $1608+656$}

\subsection{HST Observations of B1608+656}

B1608+656 was observed with the ACS camera on HST in the F606W and F814W filters in 2004 August (Proposal 10158;

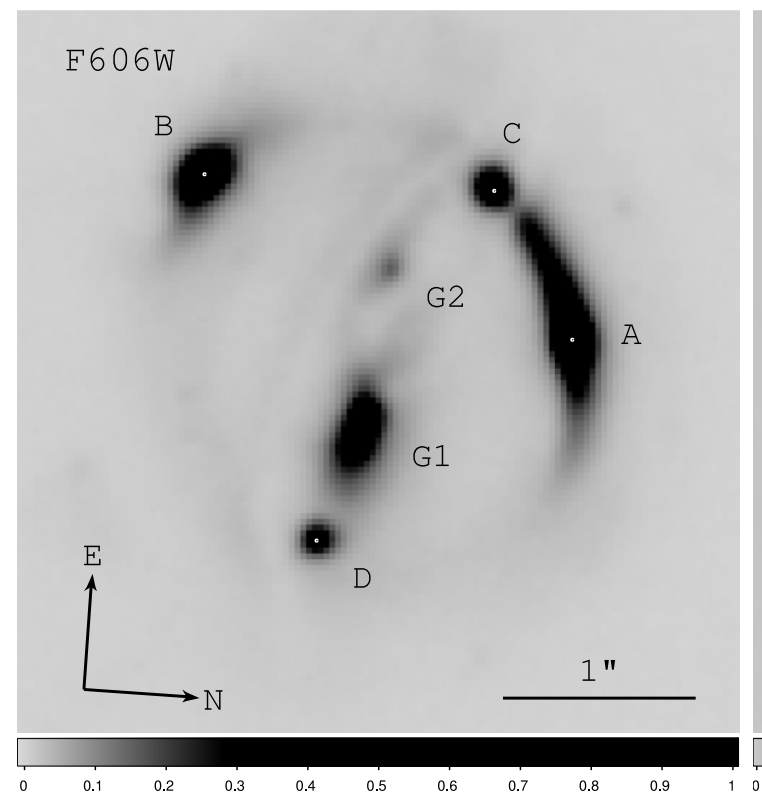

PI:Fassnacht), specifically to get high signal-to-noise ratio $(\mathrm{S} / \mathrm{N})$ images of the lensed source emission. Table 2 summarizes the observations. Each orbit of the ACS visits consisted of one four-exposure dither pattern in either F606W or F814W through the Wide Field Channel (WFC). We used the same dither pattern described in York et al. (2005) to permit drizzling to a higher angular resolution than the default ACS CCD pixel size $\left(\sim 00^{\prime} \cdot 05\right)$. This subpixel scale is especially important for characterizing the PSF.

In order to correct for the dust extinction in the lens system, we also include the Near Infrared Camera and Multi-Object Spectrometer 1 (NICMOS) F160W images (Proposal 7422; PI:Readhead). Details of the NICMOS observations are also listed in Table 2.

The ACS images of B1608+656 are presented in Figure 3 and show the two lensing galaxies and the presence of a dust lane through the system. We need to correct for both the dust

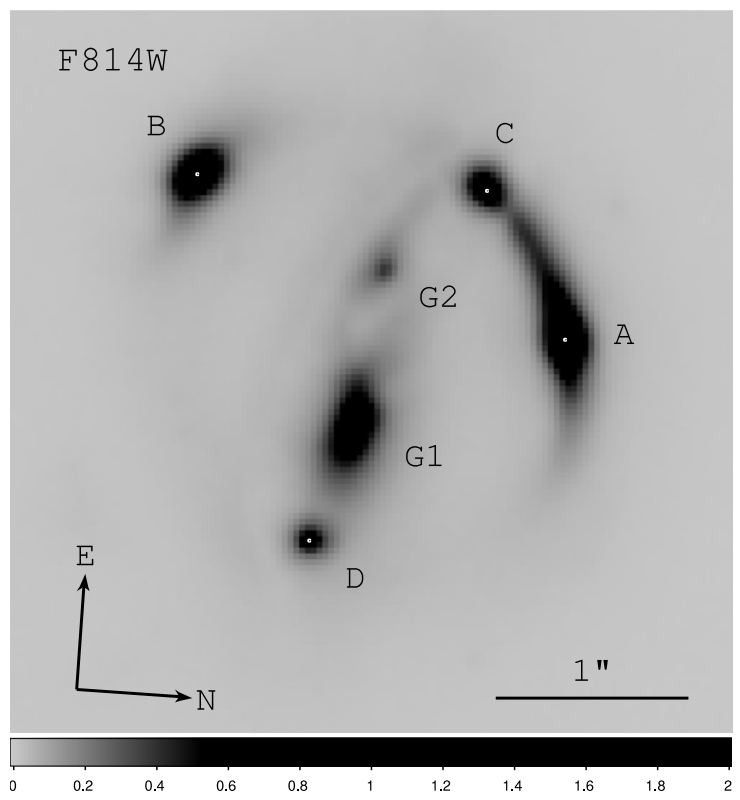

Figure 3. Left-hand (right-hand) panel: drizzled HST ACS F606W (F814W) images with 0'03 pixels from 9 (11) HST orbits. The dust lane and interacting galaxy lenses are clearly visible. The white dots indicate the centroid positions of the images. 
lane and the light from the lens galaxies, which can affect the isophotes of the Einstein ring of the extended lensed source. Before we can determine the amount of extinction, we need to first unify the resolutions of the images in different wavelength bands due to PSF dependencies. This requires PSF modeling, deconvolution, and reconvolution for images. Having unified the resolutions of the images, we can determine the intrinsic colors of the various components (lens galaxies, lensed source galaxy, AGN at the core of the source galaxy) in the system that are required for the dust correction. After correcting for dust, we can then determine the light profiles of $\mathrm{G} 1$ and $\mathrm{G} 2$ by fitting them with Sérsic profiles $\left(I(r) \propto \exp \left(-(r / a)^{1 / n}\right)\right.$, where $r$ is the radial coordinate, $a$ is a scale length, and $n$ is known as the Sérsic index; Sérsic 1968). It is only at this stage, with the PSF, dust map, and lens galaxies' light profiles, that we can recover the lensed Einstein ring surface brightness distribution for lens potential modeling.

To execute the above plan of attack, in Section 5.2, we begin by describing the drizzling process for the ACS images that are used for the analysis. In Sections 5.3-5.5, we present a suite of PSF, dust, and lens galaxies' light models and describe in detail how they are obtained. Finally, in Section 5.6, we compare these models.

\subsection{Image Drizzling}

In the following subsections, we briefly describe the drizzling process for combining the dithered ACS images and discuss the alignment of the NICMOS image to the ACS image.

\subsubsection{ACS Image Processing}

The ACS data were reduced using the multidrizzle package (Koekemoer et al. 2002) in an early version of the HAGGLeS image-processing pipeline (P. J. Marshall et al. 2009, in preparation), producing drizzled images with a $00^{\prime} 03$ pixel scale. The drizzled ACS images are shown in Figure 3. The corresponding output weight images from multidrizzle give the values for the inverse variance of each pixel. We approximate the noise covariance matrix as diagonal and use the variance pixel values for the diagonal entries, even though drizzling will correlate the noise between adjacent pixels. It is assumed that the effect of drizzling can be modeled as having a diagonal covariance matrix with the diagonal elements rescaled (Casertano et al. 2000). In practice, we do not need to do the rescaling because the ranking of the models using the relative log evidence values from the source reconstruction is insensitive to rescaling of the covariance matrix.

A pixelated representation of a continuous intensity distribution generally introduces error in the interpolated intensity values between pixels, especially for intensity distributions with sharp features. This error should be incorporated into the likelihood function. Therefore, for modeling the source-intensity distribution on a grid (in Sections 5.6 and 6), we also include the error due to pixelization on the image and source planes (which we call "regridding error") in the image covariance matrix. We express the regridding error on the image plane in terms of the data (instead of on the source plane and transforming it to the image plane) in order to obtain a noise map that is independent of the pixelated lens modeling. The regridding error associated with pixel $i$ is

$$
\left(\sigma_{\text {grid }}^{2}\right)_{i}=\frac{1}{12} \mu_{i} \frac{\Delta \beta^{2}}{\Delta \theta^{2}} \sum_{j \in \text { pixels adjacent to } i}^{N_{\text {adj }}} \frac{\left(d_{j}-d_{i}\right)^{2}}{N_{\text {adj }}}
$$

where $\mu_{i}$ is the lensing magnification at pixel $i, \Delta \beta$ is the source pixel size, $\Delta \theta$ is the image pixel size, $N_{\text {adj }}$ is the number of pixels adjacent to pixel $i$, and $d_{i}\left(d_{j}\right)$ is the image intensity at pixel $i(j)$. The summation divided by $\Delta \theta^{2}$ in the above equation is a conservative estimate on the error due to pixelization on the image plane. Since sharper features in the image have larger gradients (hence, larger values for the summations), the regridding error is higher in these areas by construction. The remaining quantities in the equation, $\mu_{i} \Delta \beta^{2} / 12$, account for the uncertainty in the predicted image (the source image mapped to the image plane) due to the pixelization of the source-intensity distribution. The factor $1 / 12$ is the second moment of a uniform distribution between -0.5 and 0.5 . When one constructs the predicted image by mapping each image pixel to the source plane and reading off the source-intensity value, the mapped source position (of an image pixel) is generally not centered on a source pixel, but have on average a $(1 / \sqrt{12})$-pixel shift from the center of the source pixel. Therefore, $\Delta \beta / \sqrt{12}$ is the effective size of the source pixel, which is then magnified by (on average) $\sqrt{\mu_{i}}$ due to lensing. In the pixelated potential reconstruction, we approximate the magnification at each image pixel (which requires the second derivative of the potential) by the value computed from the initial potential because (1) the approximation enforces the regridding error to be independent of the pixelated potential modeling and (2) the corrected potential values are obtained on an annular region only a few pixels thick. Having obtained an estimate for the regridding error, we add it in quadrature to the variance from the weight image to obtain the entries of the approximated diagonal covariance matrix.

The inclusion of the regridding error is important for sourceintensity reconstructions with sharp intensity features (such as the presence of a bright core); it has the effect of stabilizing the evidence values with respect to choices in the source pixelization. Without including the regridding error, a pixelated description of, for example, a source-intensity distribution with a bright core would be highly sensitive to the centering of the core on the source pixels. A small mismatch could create large image residuals near the cores that would veto an otherwise good lensing model, which has the rest of the extended features well described. Such an undesirable effect is mostly removed by the inclusion of the regridding error. For B1608+656, the ratio of the regridding error to the error from the multidrizzle weight image is around $\sim 30$ near the image centroids and $\sim 1$ in other parts in the Einstein ring.

\subsubsection{NICMOS Image Processing}

The NICMOS F160W image was taken from Koopmans et al. (2003). Drizzled images on rectangular grids for different instruments are generally not on the same resolution and not aligned. This is the case for the NICMOS and ACS images. We use SWarp ${ }^{13}$ to align the combined NICMOS image to the ACS images. The final SWarped NICMOS F160W image with 0'.03 pixel scales is shown in Figure 4.

\subsection{PSF Modeling}

In this subsection, we describe the procedure for obtaining the PSFs for each of the ACS and the NICMOS data sets.

\footnotetext{
13 A package developed by Emmanuel Bertin at Institut d'Astrophysique de Paris for resampling and coadding together FITS images.
} 


\subsubsection{ACS PSF}

The ACS PSF is both spatially and temporally varying (e.g., Rhodes et al. 2007). One source of temporal variation is the "breathing" of the telescope while it orbits, which causes the focal length (and, hence, the PSF) of the telescope to change. Instead of adopting a universal PSF, we take the approach of modeling several PSFs using different means, and quantitatively comparing them using the Bayesian analysis described in Section 2.2.1. This has the advantage of using the data (the observed image) to rank the models. For each of the two drizzled ACS images, we create five models for the PSF either based on the TinyTim package (Krist \& Hook 1997) or from the unsaturated stars in the field: (1) drizzled PSF ("PSF-drz") from a set of TinyTim simulations (following Rhodes et al. 2007), (2) single (nondrizzled) TinyTim PSF ("PSF-f3") with a telescope focus value of -3 , (3) the closest star ("PSF-C") located at $\sim 9$ " in the northeast direction from B1608+656 in the drizzled ACS field with a Vega magnitude of 21.3 in F814W, (4) bright star \#1 ("PSF-B1") that is located at $\sim 1$ '.9 southwest of B1608+656 in the drizzled ACS field with a Vega magnitude of 18.7 in F814W, and (5) bright star \#2 ("PSF-B2") that is located at 1'.6 south of B1608+656 in the drizzled ACS field with a Vega magnitude of 19.1 .

The TinyTim frame(s) were drizzled and resampled to pixel sizes of 0.03 to match the resolution of the ACS images. We keep in mind that the TinyTim PSFs (PSF-drz and PSF-f3) may be insufficient due to the time-varying nature of the PSF and the aging of the detector since the TinyTim code was written. We expect the closest star to B1608+656 (PSF-C) to be a good approximation to the PSF because the spatial variation of the PSF across $\sim 9^{\prime \prime}$ should be negligible and any temporal variations are the same as in the lens system. However, this closest star is not bright enough to see the secondary maxima in the PSF, so we additionally include two of the brightest stars in the drizzled field mentioned above. For each of the stars in F606W and F814W, we make a small cutout around the star $(25 \times 25$ pixels for PSF-C, $51 \times 51$ pixels for PSF-B1, and $41 \times$ 41 pixels for PSF-B2) and center it on a $200 \times 200$ grid, which is the size of the drizzled science image cutouts of B1608+656 that are used for the image processing.

\subsubsection{NICMOS PSF}

The NICMOS PSF is thought to be more stable, and thus we assume a TinyTim model for it. The output TinyTim PSF is in the CCD frame of NICMOS with pixel size 0.'043. As with the F160W science image, the PSF was SWarped to be aligned with the ACS images with 0'.03 pixels. Since there is only one PSF model for NICMOS, PSF specifications throughout the rest of this paper refer to the ACS PSFs.

\subsection{Dust Correction}

With observations in two or more wavelengths, we can correct for the dust extinction using empirical dust extinction laws. We adopt the extinction law of Cardelli et al. (1989) with the following dust extinction ratios at the redshift of the lens $z_{\mathrm{d}}=$ 0.63 for $R_{V}=3.1$ (Galactic extinction): $A_{\mathrm{F} 606 \mathrm{~W}} / A_{V}=1.56$, $A_{\mathrm{F} 814 \mathrm{~W}} / A_{V}=1.14$, and $A_{\mathrm{F} 160 \mathrm{~W}} / A_{V}=0.41$, where $A_{\lambda}$ is the extinction (difference between the observed and intrinsic magnitudes) at wavelength $\lambda$. These dust extinction ratios agree with the values from the extinction law in Pei (1992) to within $1.5 \%$. In order to correct for the extinction, we need to know the intrinsic colors of the objects (details in Section 5.4.1). For

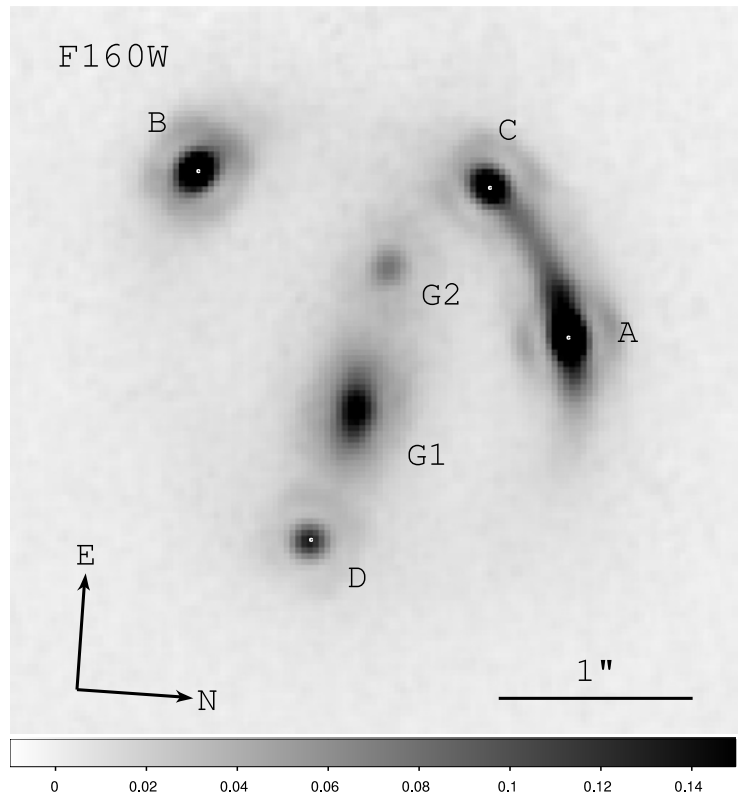

Figure 4. HST NICMOS F160W image that is SWarped to aligned to the ACS frame with a $0 . \prime 03$ pixel size. The white dots indicate the centroid positions of the images.

each color type of object (the lens galaxies, the source galaxy, and the AGN of source galaxy), we denote the intrinsic color by $Q_{F}=\left(m_{F \text {,intrinsic }}-m_{1 \text {,intrinsic }}\right)$, where $F=1, \ldots, N_{\mathrm{b}}$ is in sequence from the reddest to the bluest wavelengths (by construction $Q_{1}=0$ ), and $N_{\mathrm{b}}$ is the number of wavelength bands used for dust correction. Combining the dust extinction ratios and the definition of intrinsic colors, we can model the observed magnitudes at each image pixel in each of the wavelength bands $F$ in terms of $A_{V}$ and the intrinsic magnitude of the reddest wavelength band $m_{1 \text {,intrinsic }}$ as

$$
m_{F} \equiv m_{F, \text { observed }}=m_{1, \text { intrinsic }}+Q_{F}+A_{V} k_{F}+n_{F},
$$

where $k_{F} \equiv A_{F} / A_{V}$ are constants given by the extinction law and $n_{F}$ is the noise in the data of wavelength band $F$. We can solve for $A_{V}$ and $m_{1 \text {,intrinsic }}$ at each image pixel by minimizing the following $\chi_{\text {dust }}^{2}$ for each pixel:

$$
\chi_{\text {dust }}^{2}=\sum_{F=1}^{N_{\mathrm{b}}}\left(m_{F}-m_{1, \text { intrinsic }}-Q_{F}-A_{V} k_{F}\right)^{2} .
$$

We have weighted the images of the different bands equally because the uncertainty associated with $m_{F}$ is negligible compared to that of $Q_{F}$, and the uncertainties in $Q_{F}$ are of comparable magnitudes for the different bands $F$ relative to the reddest. The solution that minimizes $\chi_{\text {dust }}^{2}$ is

$$
\begin{aligned}
A_{V}= & {\left[\frac{1}{N_{\mathrm{b}}}\left(\sum_{F} k_{F}\right)\left(\sum_{F} m_{F}\right)\right.} \\
& -\frac{1}{N_{\mathrm{b}}}\left(\sum_{F} k_{F}\right)\left(\sum_{F} Q_{F}\right) \\
& \left.-\sum_{F} k_{F} m_{F}+\sum_{F} k_{F} Q_{F}\right] / \\
& {\left[\frac{1}{N_{\mathrm{b}}}\left(\sum_{F} k_{F}\right)^{2}-\sum_{F} k_{F}^{2}\right], }
\end{aligned}
$$



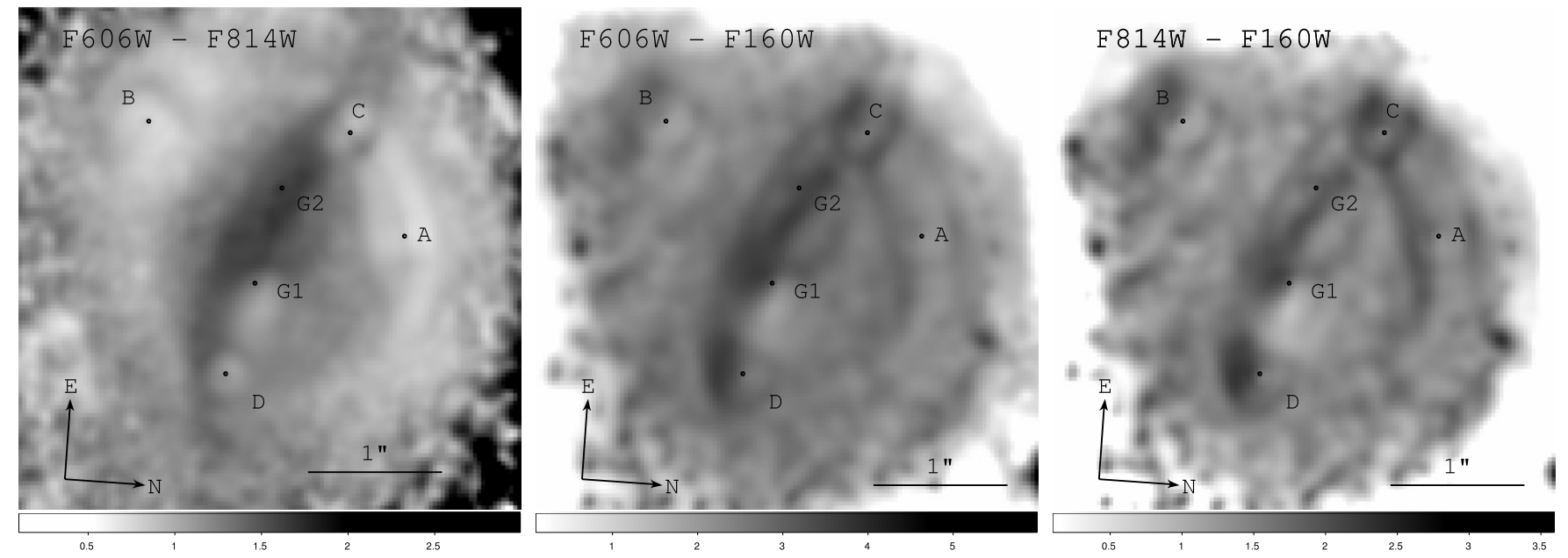

Figure 5. From left to right: the derived color maps F606W-F814W, F606W-F160W, and F814W-F160 using PSF-B1.

and

$$
m_{1, \text { intrinsic }}=\frac{1}{N_{\mathrm{b}}}\left(\sum_{F} m_{F}-\sum_{F} Q_{F}-\sum_{F} A_{V} k_{F}\right),
$$

where the sums over $F$ go from $1, \ldots, N_{\mathrm{b}}$. We emphasize that Equations (18) and (19) give the $A_{V}$ and $m_{1, \text { intrinsic }}$ at each pixel. Since $A_{V}$ varies from pixel to pixel (depending on the amount of dust seen in that pixel), the various $A_{V}$ values of all pixels provide a dust map. Similarly, the $m_{1 \text {,intrinsic values of all }}$ pixels give the dust-corrected image in the reddest wavelength band. The resulting values of $m_{1 \text {,intrinsic }}$ and the intrinsic colors yield the intrinsic (dust-corrected) magnitudes in the other bands $m_{F \text {,intrinsic }}$, where $F=2, \ldots, N_{\mathrm{b}}$. For any one band $F$, we can then construct the diagonal dust matrix $\mathbf{K}$ in Equation (13) whose nonzero entries are $10^{-0.4 A_{V} k_{F}}$.

\subsubsection{Obtaining the Intrinsic Colors}

The dust correction method outlined above requires the intrinsic colors to be determined from the color maps. To construct the color maps, we need to unify the different resolutions of the images in different bands (due to the wavelength dependence of the PSF). We do so by deconvolving the F606W, F814W, and F160W images using their corresponding PSFs, and reconvolving the images with the F814W PSF for each set of the five ACS PSFs and the single NICMOS PSF described in Section 5.3. Reconvolved images are preferred to deconvolved images, because the latter show small-scale features (of a few pixels' size) that are artificial due to the amplification of the noise during the deconvolution process. We select the F814W PSF for the reconvolution because $\mathrm{F} 814 \mathrm{~W}$ will be used for the lens potential modeling, due to its high $\mathrm{S} / \mathrm{N}$ compared with F160W and its less severe dust extinction compared with F606W. In working with the reconvolved images, we assume that the dust varies on a scale larger than the F814W PSF, which is true for the regions near the Einstein ring. For the deconvolution, we use IDL's max_entropy iterative routine that is based on the algorithm by Hollis et al. (1992). We were unable to deconvolve the ACS F814W image using PSF-f3. This suggests that PSF-f3 is a bad model, which we have expected due to temporal variations in the PSF. PSF-f3 is a single-epoch PSF whereas the F814W image was drizzled from multiple exposures. We, therefore, discard this PSF model.
For each set of PSF models (PSF-drz, PSF-C, PSF-B1, and PSF-B2 for ACS, and TinyTim PSF for NICMOS), we construct the color maps F606W-F814W, F606W-F160W, and F814W-F160W from the reconvolved F606W, F814W, and F160W images, respectively. Figure 5 shows the three color maps derived for PSF-B1. Regions with bluer color slightly west of G1 are shown in all three color maps. Since the centroid of this blue region is offset from the centroid of $\mathrm{G} 1$, we believe that this blue region arises from differential reddening and not from intrinsic color variations within $\mathrm{G} 1$, which is an elliptical galaxy (Surpi \& Blandford 2003). Since elliptical galaxies typically contain little dust, Koopmans \& Fassnacht (1999) and Surpi \& Blandford (2003) suggested that the dust comes from G2, likely a dusty late-type galaxy, through dynamical interaction. This may explain why the spectrum of G1 shows signatures of a young stellar population plus a poststarburst population (Dressler \& Gunn 1983; Myers et al. 1995; Surpi \& Blandford 2003; Koopmans et al. 2003): gas from G2 may have been transferred to $\mathrm{G} 1$, where the tidal interactions may have triggered star formation.

The color maps also show regions of bluer color around images $\mathrm{C}$ and $\mathrm{D}$, and we again believe that these are mostly differential reddening due to the misalignment of the image positions and the centroids of these blue regions, especially in F606W-F160W and F814W-F160W. Furthermore, we find more dust at the crossing point of the isophotal separatrix (the figure-eight-shaped intensity contour) of the image pair $\mathrm{A}-\mathrm{C}$. This is encouraging, as lensing models indeed predict the crossing point to be closer to image A (see discussion in Section 5.4.2). However, these bluer regions near images $C$ and $\mathrm{D}$ may also arise from the lensed source being intrinsically bluer than the surrounding emission. The F814W-F606W color for these blue regions is consistent with typical star-forming galaxies (e.g., Coleman et al. 1980). In the F606W-F814W color map, there is a faint ridge of redder color connecting images $\mathrm{A}$ and $\mathrm{C}$. This may be due to the asymmetry in the stellar PSF model (with the star position not exactly centered within a pixel), which would cause the F606W and F814W isophotes to shift relative to each other after the deconvolution and reconvolution. For the color maps from the other PSF models, we find that the color maps from PSF-C and PSF-B2 look similar to that from PSF-B1 with varying amounts of noise due to varying brightnesses of the stellar PSFs. PSF-drz gave color maps that differ from those from the stellar PSFs (PSF-C, PSF-B1, 

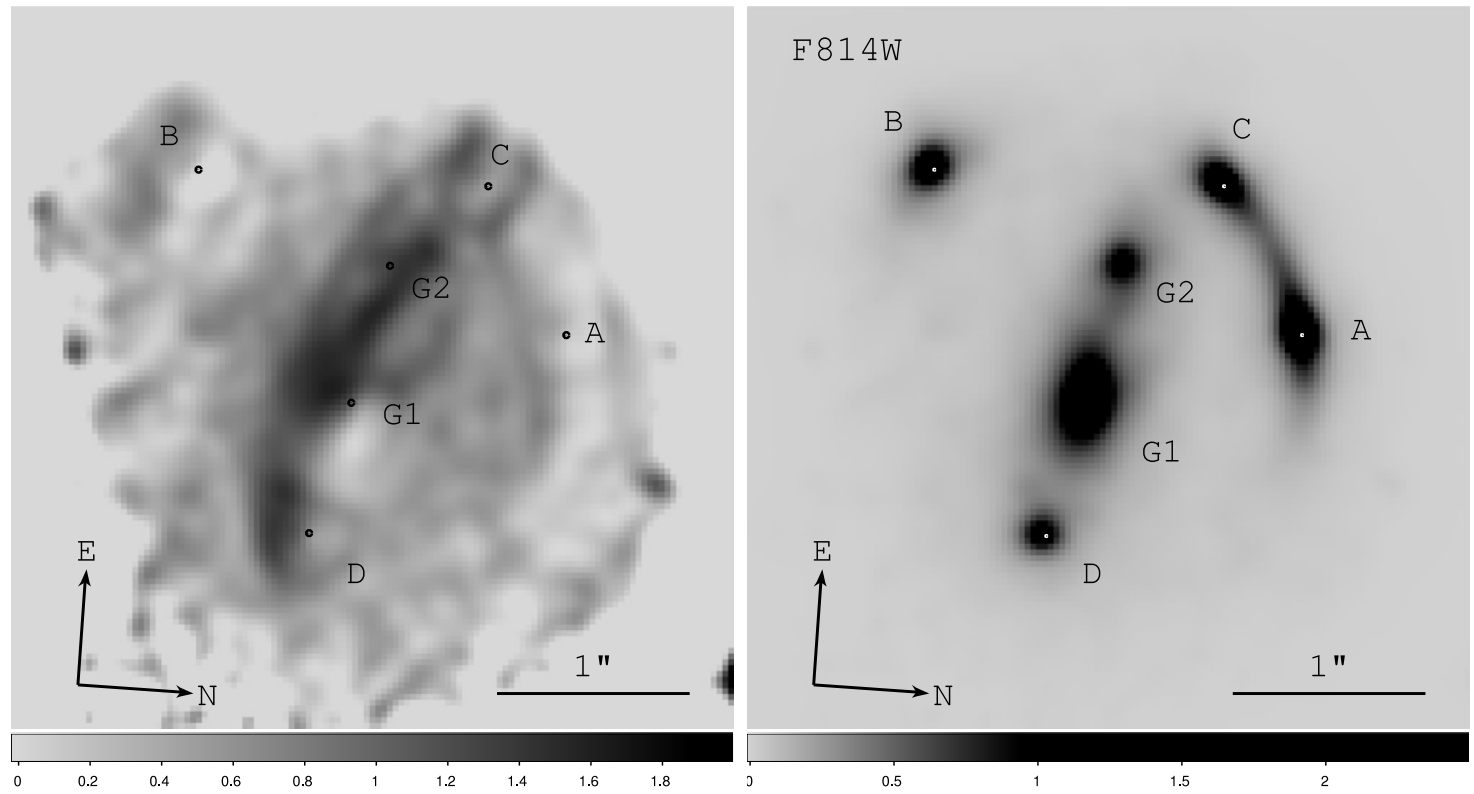

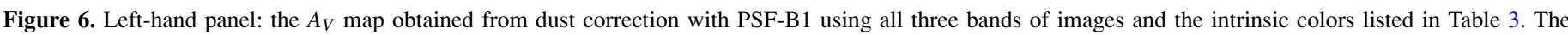

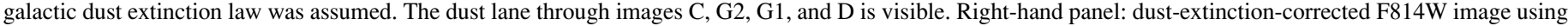

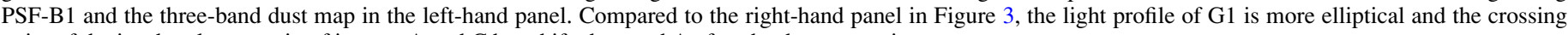
point of the isophotal separatrix of images $\mathrm{A}$ and $\mathrm{C}$ has shifted toward $\mathrm{A}$ after the dust correction.

and PSF-B2) because PSF-drz, especially in the F606W band, did not exhibit a single brightness peak but a string of equal brightness pixels at the center due to frame alignment difficulties during the drizzling process. This caused the brightest pixels in the Einstein ring to shift by $\sim 1$ pixel after the deconvolution and the reconvolution process in $\mathrm{F} 606 \mathrm{~W}$, and created artificial sharp highlights tracing the edge of the ring in the F606W-F814W color map. As will be seen in Section 5.6, this leads to PSF-drz and its resulting dust map giving a lower goodness of fit in the lens inversion, and hence being ranked lower compared with other models.

In each of the color maps, we define three color regions for the three color components: one within the Einstein ring for the lens galaxies (we assume G1 and G2 to have the same colors), one for the Einstein ring of the lensed extended source, and one for the lensed AGN (core of the extended source). Following Koopmans et al. (2003), we determine the bluest color within each region, assume that this part of the region was not absorbed by dust, and adopt this color as the intrinsic color. This assumes that each of the three components has a constant intrinsic color. This would allow us to obtain the differential reddening for each of the components across the lensed image; absolute reddening is not needed because a uniform dust screen does not affect lens modeling. Table 3 lists the intrinsic colors for each of the three pairs of color maps. The intrinsic colors of F606W-F814W are not identical to the difference between F606W-F160W and F814W-F160W, but agree within the uncertainties $(0.02-0.1)$.

\subsubsection{Resulting Dust Maps}

With the intrinsic colors determined for each PSF model, we obtain two dust maps ( $A_{V}$ maps) using (1) only the ACS F606W and F814W images and (2) the ACS F606W and F814W images together with the NICMOS F160W image. In this way, we can assess whether the inclusion of the lower S/N NICMOS image (with the much broader PSF) improves the dust correction.

The left-hand panel of Figure 6 is the resulting $A_{V}$ dust map derived using PSF-B1 and using images in all three bands. The

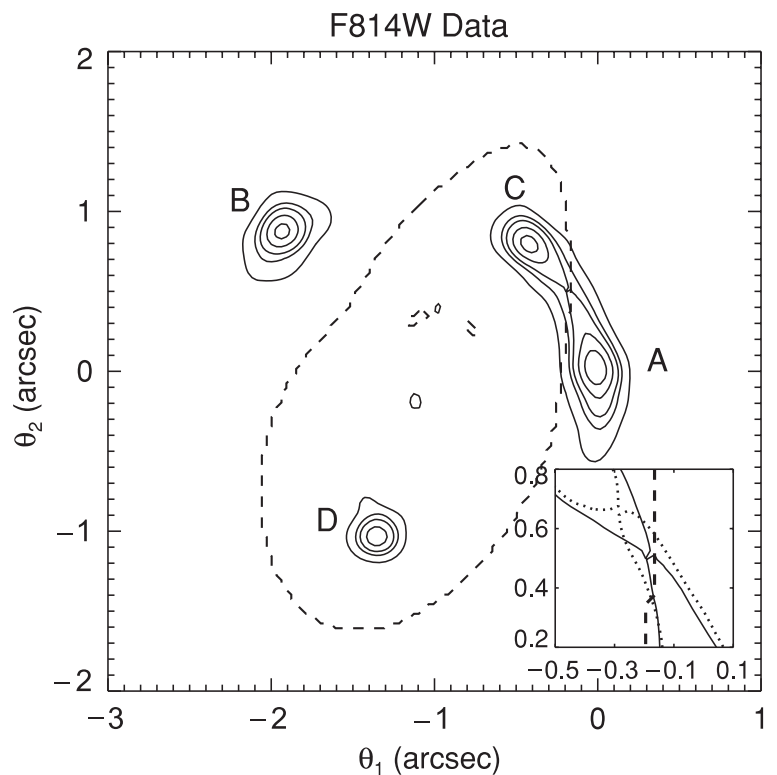

Figure 7. Crossing isophotes of the B1608+656 Einstein ring. Shown here is the dust-corrected and galaxy-subtracted F814W image (solid contours), with the critical curves of the SPLE1+D (isotropic) potential model (Koopmans et al. 2003) overlaid (dashed curves). The inset shows a "zoomed-in" view of the region between images $\mathrm{C}$ and $\mathrm{A}$; here, the dotted curves in the zoomed-in panel are the intensity contours of the galaxy-subtracted F814W image without dust correction. After dust correction, the crossing point of the isophotal separatrix (the center of the figure-eight isophote) is shifted toward the critical curve, indicating successful dust correction.

dust map shows the east-west dust lane through the system (absorbing light from $\mathrm{C}, \mathrm{G} 2, \mathrm{G} 1$, and $\mathrm{D}$ ) that is visible in the original drizzled ACS F606W and F814W images. There is little extinction near images $\mathrm{A}$ and $\mathrm{B}$, but there are faint rings surrounding the images that are mostly due to imperfect F160W deconvolution. We note that the low $\mathrm{S} / \mathrm{N}$ exterior to the Einstein ring results in the dust map being noisy in this area. We make sure that these noisy areas are not included in the Bayesian 

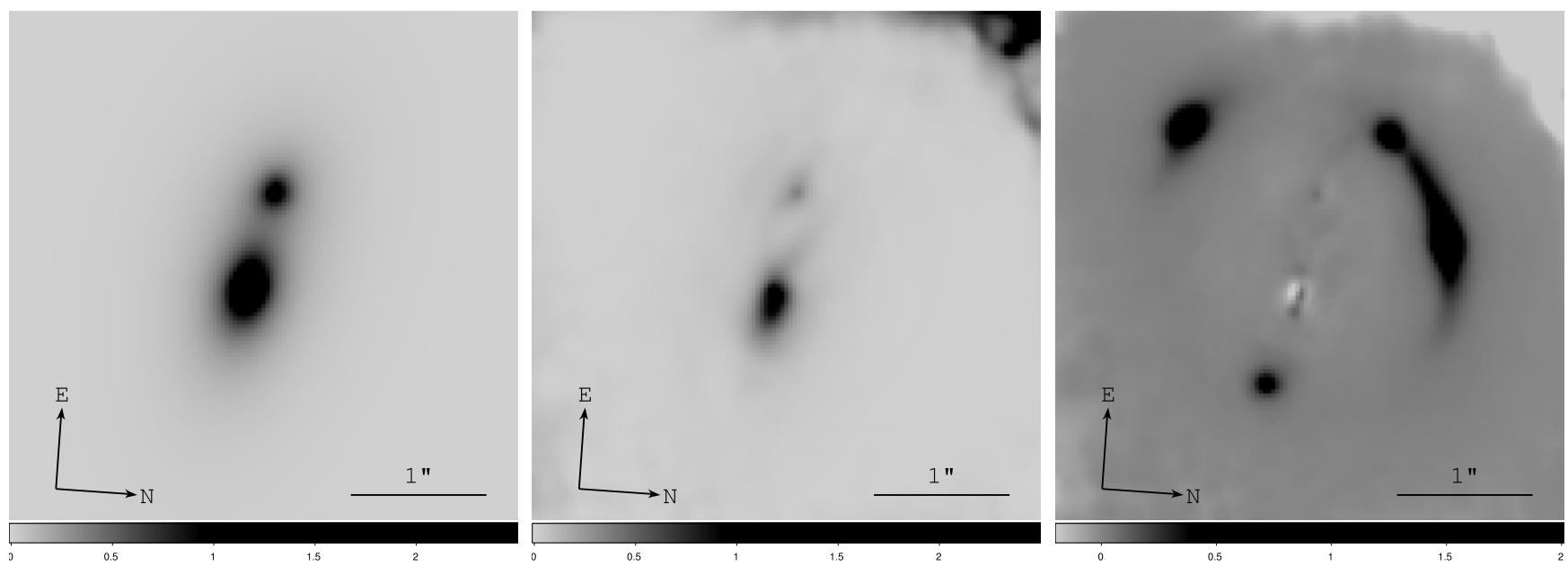

Figure 8. Sérsic lens galaxy light profile fitting to the dust-corrected F814W image, with PSF-B1 and its corresponding three-band dust map, using GALFIT. The left-hand panel shows the best-fit Sérsic light profiles with Sérsic indices $\left(n_{\mathrm{G} 1}, n_{\mathrm{G} 2}\right)=(3,4)$. The middle panel shows the dust-extincted galaxy light profiles, which is the left-hand panel with the dust extinction added back in. The right-hand panel shows image residual (difference between the F814W drizzled image in Figure 3 and the middle panel) with misfit near the cores of the lens galaxies of $\sim 25 \%-35 \%$.

Table 3

Intrinsic Colors of the AGN, Einstein Ring, and Lens Galaxies in B1608+656

\begin{tabular}{llccc}
\hline \hline & & F606W-F814W & F814W-F160W & F606W-F160W \\
\hline PSF-drz & AGN & 0.50 & 1.4 & 1.91 \\
& Ring & 0.70 & 1.5 & 2.20 \\
& Lens & 0.84 & 1.0 & 1.88 \\
PSF-C & AGN & 0.78 & 1.3 & 2.10 \\
& Ring & 0.84 & 1.5 & 2.30 \\
& Lens & 1.04 & 1.0 & 2.05 \\
PSF-B1 & AGN & 0.72 & 1.1 & 1.85 \\
& Ring & 0.76 & 1.3 & 2.10 \\
& Lens & 1.04 & 0.82 & 1.85 \\
PSF-B2 & AGN & 0.70 & 1.17 & 1.99 \\
& Ring & 0.80 & 1.3 & 2.10 \\
& Lens & 1.01 & 0.85 & 1.92 \\
\hline
\end{tabular}

Notes. The intrinsic colors are based on color maps derived from the four ACS PSF models (PSF-drz (drizzled TinyTim), PSF-C (closest star), PSF-B1 (bright star \#1), and PSF-B2 (bright star \#2)) and the single NICMOS TinyTim PSF. The intrinsic colors for each of the three color regions are determined from the bluest colors in the respective region. The uncertainties on the intrinsic colors vary from 0.02 to 0.1 . The higher uncertainties are associated with the F160W image, which has a lower S/N.

evidence computations in Sections 5.6 and 6. The right-hand panel of Figure 6 is the resulting dust-corrected F814W image that exhibits two signs of proper dust correction: the correctly shifted crossing point of the isophotal separatrix of the image pair A-C, as shown more clearly in Figure 7, and the smoother lens galaxy profiles. As a result of recovering the absorbed light, the dust-corrected image has higher intensity values than the uncorrected image. Therefore, we create a weight map for the dust-corrected image by scaling the multidrizzle weight image in order to keep the $\mathrm{S} / \mathrm{N}$ of each pixel the same (before and after dust correction). This "dust-corrected weight image" will be used in the next section for determining the lens galaxy light.

The dust maps obtained from the other PSF models with or without the inclusion of the NICMOS image show similar features except for the following two dust maps.

1. The ACS-only (no NICMOS) dust map from PSF-B2 showed a faint ridge of dust connecting images $\mathrm{A}$ and $\mathrm{C}$. As explained, this may be due to the asymmetrical/bad PSF model. Since the dust map otherwise exhibits the correct features, we keep this dust map for the next analysis step.
2. The ACS-only dust map from PSF-drz showed prominent artificial lensing arc features due to the $\sim 1$ pixel offset in the image positions/arcs in the deconvolved and reconvolved F606W and F814W images, respectively. Therefore, we discard this dust map of the ACS-only images for PSF$\mathrm{drz}$, but keep the dust map derived from using all three bands (that includes NICMOS).

After discarding the ACS PSF-f3 and the ACS-only dust map from PSF-drz, we have a total of seven dust maps (and resulting dust-corrected F814W images). All of these are reasonable dust corrections to use since they are derived using representative PSFs and intrinsic colors. We will compare these dust maps and PSF models in Section 5.6.

\subsection{Lens Galaxy Light}

For each of the seven resulting dust-corrected F814W images in Section 5.4.2 and its corresponding PSF, we create an elliptical mask for the lens galaxies' region that excludes the Einstein ring and fit the lens galaxies' light to elliptical Sérsic profiles using GALFIT (Peng et al. 2002). In particular, we impose the Sérsic indices to be one of the following pairs: $\left(n_{\mathrm{G} 1}, n_{\mathrm{G} 2}\right)=(1,1),(2,2),(3,3),(3,4),(4,3),(4,4)$. There are more pairings with $n=3$ and $n=4$ since previous works by, for examples, Blandford et al. (2001) and Koopmans et al. (2003) found G1 to be well described by $n=4$ (de Vaucouleurs profile). With the dust-corrected weight image, we obtain a reduced $\chi^{2}$ value for each of the profile fittings. For each dustcorrected F814W image, we pick the Sérsic index pair with the lowest reduced $\chi^{2}$ from the fit (top two pairs in the case of PSF-drz) and list it in Table 4. As an illustration, Figure 8 shows the GALFIT Sérsic $\left(n_{\mathrm{G} 1}, n_{\mathrm{G} 2}\right)=(3,4)$ results of the dust-corrected F814W image using the three-band dust map from PSF-B1. The dark (light) patches in the upper righthand corner of the middle (right-hand) panel result from the noisy dust map due to low signal to noise in this area. Apart from this area and the lens galaxies' cores, most of the observed lens galaxies' light matches the dusted Sérsic profiles in the middle panel, as shown in the residual map in the right-hand panel. The misfit near the cores could be due to intrinsic color variations in the lens galaxies, the dust screen assumption, PSF imperfections, and/or inapplicability 
Table 4

Best-Fitting Sérsic Light Profiles for the Lens Galaxies G1 and G2 for the Seven Different Dust-Corrected F814W Images Based on Different PSFs and Dust Maps

\begin{tabular}{lccc}
\hline \hline PSF & Dust Map & Sérsic Indices $\left(n_{\mathrm{G} 1}, n_{\mathrm{G} 2}\right)$ & Reduced $\chi_{\text {lens light }}^{2}$ \\
\hline drz & Three-band & $(3,4)$ & 4.48 \\
drz & Three-band & $(3,3)$ & 4.53 \\
C & Three-band & $(3,4)$ & 5.11 \\
C & Two-band & $(3,3)$ & 6.13 \\
B1 & Three-band & $(3,4)$ & 5.53 \\
B1 & Two-band & $(2,2)$ & 7.16 \\
B2 & Three-band & $(2,2)$ & 5.95 \\
B2 & Two-band & $(2,2)$ & 8.19 \\
\hline
\end{tabular}

Notes. In the PSF column, "drz" = drizzled TinyTim, "C" = closest star, "B1" = bright star \#1, and "B2" = bright star \#2. In the dust map column, "two-band" represents the dust map obtained from just the two ACS bands, and "three-band" represents the dust map obtained from the two ACS and the one NICMOS bands.

of a single Sérsic model at the center. Nonetheless, accurate light fitting near the cores of the lens galaxies is not important; it is for the isophotes of the Einstein ring that we need to have accurate dust and lenses' light corrections for the lens modeling. For the ring, the dust screen assumption in our approach is valid.

\subsection{Comparison of PSF, Dust, and Lens Galaxy Light Models}

Following the method outlined in Section 4, we can use the Bayesian evidence from the source-intensity reconstruction to compare the different PSF (B), dust (K) and lens galaxy light $(\boldsymbol{l})$ models. For each set of $\mathbf{B}, \mathbf{K}$, and $\boldsymbol{l}$, we obtain the corresponding galaxy-subtracted $\mathrm{F} 814 \mathrm{~W}$ image $(\boldsymbol{d}-\mathbf{B} \cdot \mathbf{K} \cdot \boldsymbol{l})$ that is analogous to the one shown in the right-hand panel of Figure 8 . We then make a $130 \times 130$ pixel cutout of the 0.03 galaxy-subtracted image and use the SPLE1+D (isotropic) lens potential model in Koopmans et al. (2003), which is the most up-to-date simply parameterized lens potential model for B1608+656, for the source-intensity reconstruction. Due to the source and image pixelizations, we include the regridding error (described in Section 5.2.1) in the image covariance matrix.

We select an annular region enclosing the Einstein ring and use the data inside this region for the source-intensity reconstructions for each set of the PSF, dust, and lens galaxy light models. The source grid, which we fix to have $32 \times 32$ pixels, has pixel sizes that are $\sim 0$.'022 to cover the marked elliptical annular region when mapped to the image plane. This is sufficient for achieving reasonable reconstructions and is computationally manageable. In the inversions, we reduced the PSF to $15 \times 15$ pixels to keep the matrices such as B reasonably sparse for computing speed. We try three forms of regularization: zeroth-order, gradient and curvature (e.g., Appendix A of Suyu et al. 2006).

Table 5 lists the suite of PSF, dust, and lens galaxy light models we obtained in the previous section. We label the different models by numbers from 1 to 11 in the left-most column. Models 9 and 10 correspond to the mixing of the dust maps and lens galaxy light profiles derived from PSFB1 with PSF-C and vice versa. Model 11, which is included as a consistency check, uses PSF-B1 and has no dust correction applied. For each set of models, the source-intensity distribution for B1608+656 is reconstructed. As an example, Figure 9 shows the results of the source reconstruction with gradient regularization using PSF-B1, its corresponding three-band dust
Table 5

PSF, Dust, and Lens Galaxies' Light Model Comparison Based on Bayesian Source Inversion

\begin{tabular}{rccccc}
\hline \hline & PSF & Dust Map & Sérsic $\left(n_{\mathrm{G} 1}, n_{\mathrm{G} 2}\right)$ & Reg. Type & $\begin{array}{c}\text { Log Evidence } \\
\left(\times 10^{4}\right)\end{array}$ \\
\hline 1 & drz & Three-band & $(3,4)$ & grad & 1.49 \\
2 & drz & Three-band & $(3,3)$ & grad & 1.48 \\
3 & C & Three-band & $(3,4)$ & grad & 1.60 \\
4 & C & Two-band & $(3,3)$ & zeroth & 1.40 \\
5 & B1 & Three-band & $(3,4)$ & grad & 1.56 \\
6 & B1 & Two-band & $(2,2)$ & zeroth & 1.10 \\
7 & B2 & Three-band & $(2,2)$ & grad & 1.55 \\
8 & B2 & Two-band & $(2,2)$ & zeroth & 1.23 \\
9 & C & B1/three-band & $(3,4)$ & zeroth & 1.56 \\
10 & B1 & C/two-band & $(3,3)$ & zeroth & 1.36 \\
11 & B1 & $\ldots$ & $(3,4)$ & zeroth & 1.27 \\
\hline
\end{tabular}

Notes. For each set of the PSF, dust, and lens galaxy light profiles derived in Sections 5.3-5.5, the Bayesian log evidence value is from the sourceintensity reconstruction using the SPLE1+D (isotropic) model in Koopmans et al. (2003). The uncertainty in the log evidence value due to source pixelization is $\sim 0.03 \times 10^{4}$. In the PSF column, "drz"=drizzled TinyTim, "C"=closest star, "B1"= bright star \#1, and "B2"=bright star \#2. In the dust map column, we list "two-band" for the dust map obtained from just the two ACS bands and "threeband" for the dust map obtained from the two ACS and the one NICMOS bands. Unless otherwise indicated in the dust map column, the PSF model used for the dust map derivation was the same as the corresponding PSF model in the PSF column that was used for source reconstruction. For completeness, we restate the Sérsic indices in Table 4 in the lens galaxy light profile column, which were obtained for the corresponding dust maps and PSFs specified in the dust map column. The column of "Reg. Type" refers to the preferred type of regularization for the source reconstruction, based on the highest Bayesian evidence value. It can be one of three types: zeroth-order, gradient, or curvature.

map, and the resulting Sérsic $\left(n_{\mathrm{G} 1}, n_{\mathrm{G} 2}\right)=(3,4)$ galaxy light profile. The top left-hand panel shows the reconstructed source-intensity distribution that is approximately localized, an indication that the lens potential model is close to the true potential model. In the top-middle panel, the pixels that are far from the source but are inside the caustics have lower $1 \sigma$ error values than the pixels outside the caustics due to higher image multiplicity inside the caustics. The bottom right-hand panel shows significant image residuals (the reduced $\chi^{2}$ is 1.9 inside the annulus), a sign that the PSF, dust, lens galaxy light, and/or the lens potential models are not optimal. In Section 6, we will use the pixelated potential correction scheme, which is more suitable for interacting galaxy lenses, to improve the simply parameterized SPLE1+D (isotropic) model.

The source-intensity reconstructions using other PSF and lens galaxy light models with three-band dust maps give overall similar inverted source intensities and image residuals, but the source intensities can be more or less localized and the magnitude and structures of the image residuals vary for different model sets. However, the source-intensity reconstructions using models with two-band dust maps result in source intensities that are not localized, and the image residuals show surpluses of light in the ring region and deficits of light in the lens galaxy region (corresponding to the color regions we marked for obtaining the intrinsic colors). The reason is that with only two bands, the resulting dust-corrected F814W image is highly sensitive to relative shifts between the F606W and F814W images (due to an imperfect PSF model, deconvolution, and reconvolution) and errors in the modeled intrinsic colors. The abrupt change in the modeled intrinsic colors across the boundaries of the color regions creates artificial surpluses or deficits of dust- 
Inverted Source

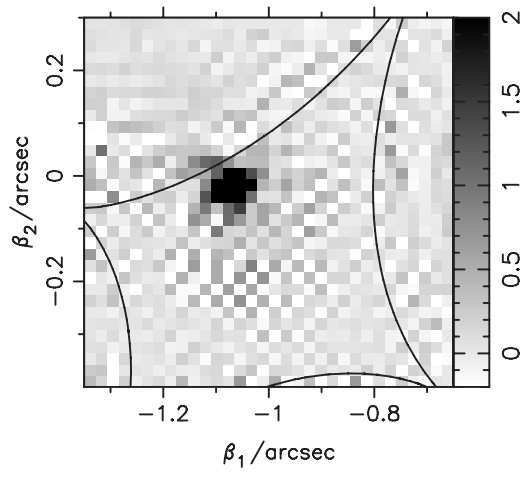

Data

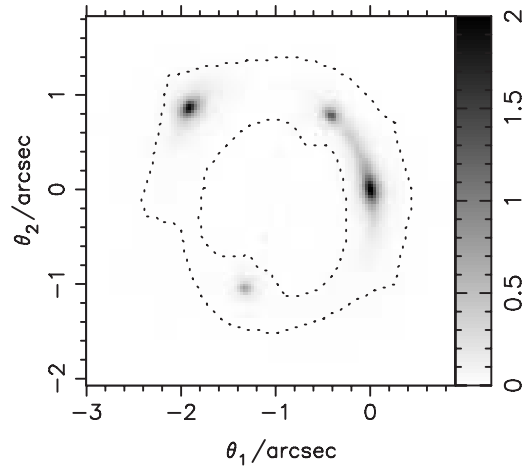

$1 \sigma$ Error of Inverted Source

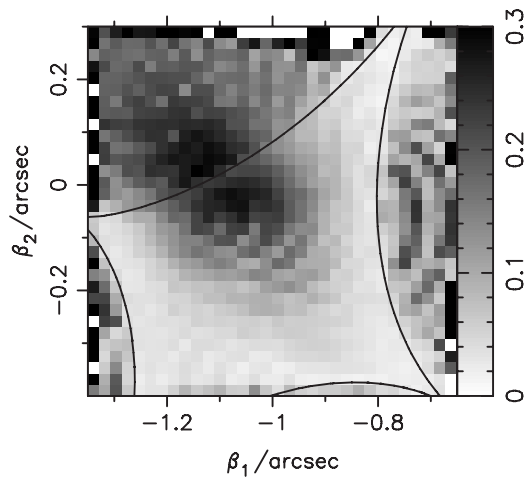

Predicted Data

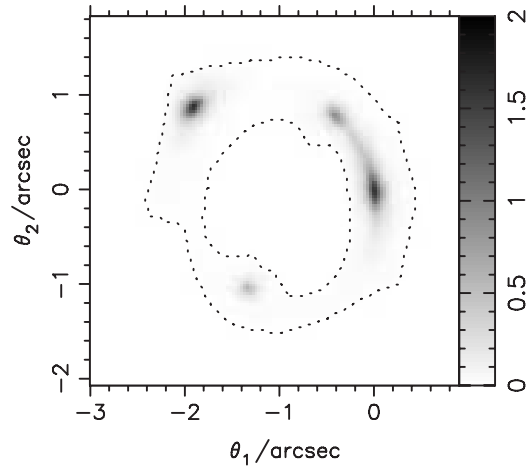

S/N Ratio of Inverted Source

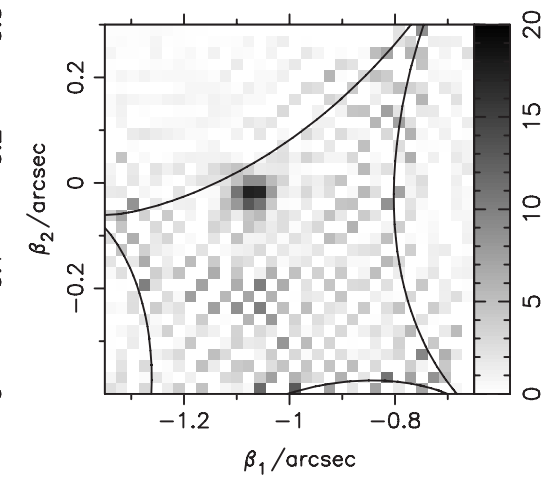

Normalized Image Residual

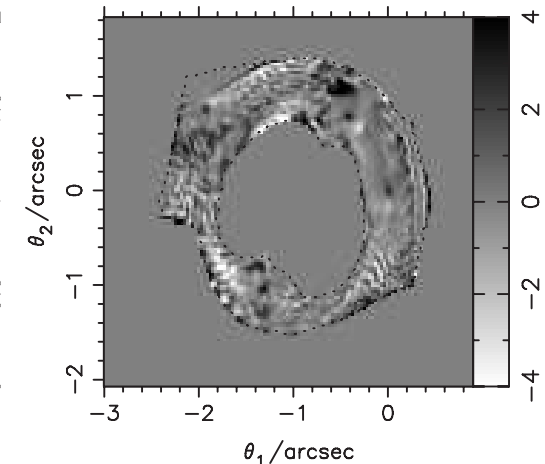

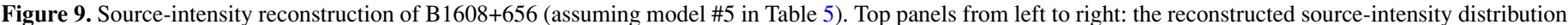

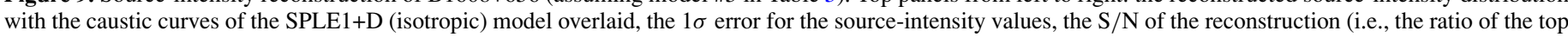

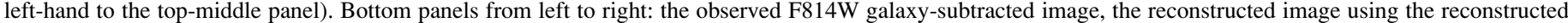

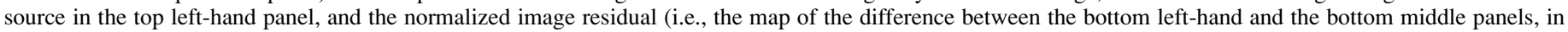
units of the estimated pixel uncertainty from the data image covariance matrix).

corrected light near the boundaries. This effect is suppressed with the addition of the F160W image because the F160W image suffers relatively little extinction, and the error due to misalignment in the images and abrupt change in the modeled intrinsic colors is reduced when one has more than two bands. A few tests suggest that the error in the dust-corrected image due to the range of intrinsic colors listed in Table 3 overwhelms the error associated with the foreground dust screen assumption for the lens galaxy light.

The source-intensity reconstruction in Model 11 with no dust correction shows significant image residuals in the extended ring, with overall surpluses of light surrounding images A and $B$ and deficits surrounding images $C$ and $D$. The source intensity is also poorly reconstructed, being nonlocalized and noisy. This illustrates the importance of dust correction for the initial SPLE1+D (isotropic) model.

\subsubsection{Results of Comparison}

Table 5 summarizes the results of model comparison. The "Reg. Type" column denotes the preferred type of regularization for the source reconstruction based on the highest Bayesian evidence value (Suyu et al. 2006). It can be one of the three types that we use: zeroth-order, gradient, and curvature. The last column lists the log evidence values from the inversions. Assuming the different models to be equally probable a priori, we use these evidence values for model comparison. The $\log$ evidence values range from $1.1 \times 10^{4}$ to $1.6 \times 10^{4}$ with uncertainties of $\sim 0.03 \times 10^{4}$ due to the finite source resolution.

The list shows that the three-band dust models have higher evidence values than the two-band dust models. This is attributed to the two-band dust models showing image residuals from the aforementioned artificial surpluses and deficits of light in the dust-corrected image. The inclusion of the NICMOS F160W image to the ACS images (F606W and F814W) for the dust correction is, therefore, crucial due to (1) the proximity in the wavelengths of the ACS images and (2) the reduction in the error associated with image misalignments and simplistic intrinsic color models.

The three-band dust models also have higher evidence values than the no-dust model. This further validates the three-band dust correction, as already indicated by Figure 7 . The evidence value of the no-dust model is in midst of the values for the two-band dust models, suggesting that the systematic effects in the two-band dust maps are comparable to the corrections that the dust maps are meant to achieve, thus leading to little improvement in the lens modeling.

The difference between the evidence values in Models 1 and 2 (where the models only differ in the Sérsic light profiles) is, in general, smaller than the difference between one of these two models and another PSF/dust model. Therefore, the source reconstruction (part of lens modeling) seems to be less sensitive to the galaxy light profiles than the PSF/dust models. This is in agreement with our finding that the dust-corrected image depends more on the PSF and the intrinsic color models than on the form of the lens galaxy light and the dust associated with the lens galaxies. Models 1 and 2 with PSF-drz have log evidence values on the low side of the collection of models with three-band dust maps, which was expected with PSF-drz not having a single brightness central peak due to misalignments in the drizzling process. The other models with three-band dust 
maps (Models 3, 5, 7, and 9) have effectively the same evidence values within the uncertainties. The models with two-band dust maps (Models 4, 6, 8, and 10) lead to a range of evidence values with the PSF-C dust map being preferred to the PSF-B1 and PSF-B2 dust maps. The two-band dust maps suggest that the shape of the primary maximum in the PSF is more important in the modeling than the inclusion of secondary maxima since PSF-C, which we expect to have a more accurate shape for the primary PSF maximum than PSF-B1 and PSF-B2, does not have the secondary maxima whereas PSF-B1 and PSF-B2 do. The asymmetry in the PSF due to the star not being centered on a single pixel may also explain the less-preferred PSF-B1 and PSF-B2. The distinction between the various stellar PSFs vanishes with the three-band dust maps, possibly due to the higher amount of noise in the three-band dust map with the inclusion of the lower S/N NICMOS image. In this case, the effects of the PSF variations across the field are suppressed.

All models preferred either the zeroth-order or gradient form of regularization, but never the curvature form; however, we mention that the difference in the log evidence values between the different regularization schemes $\left(\lesssim 3 \times 10^{2}\right)$ are on the order of the uncertainties due to source pixelization, and the resulting reconstructions for different types of regularizations are almost identical. This is because differences in evidence values between models are currently dominated by changes in goodness of fit rather than subtle differences between the prior forms. Only when the image residual is reduced will the prior (regularization) begin to play a greater role in avoiding the reconstruction to fit to noise in the data by keeping the source model simple.

This section has illustrated a method of creating sensible PSF, dust, and lens galaxy light models for the gravitational lens B1608+656. We have obtained a representative sample of models, and have compared these models quantitatively. This collection of PSF, dust, and lens galaxy light models leads to image residuals that cannot be beaten down further unless we improve the SPLE1+D (isotropic) simply-parameterized lens potential model by Koopmans et al. (2003) to take into account the two interacting galaxy lenses. The pixelated potential reconstruction of B1608+656 is the subject of Section 6.

\section{PIXELATED LENS POTENTIAL OF B1608+656}

We reconstruct the lens potential for each set of the PSF, dust, and lens galaxies' light in Models 2-11 in Table 5. We describe in detail the potential reconstruction using Model 5, which is one of the four models that, within the uncertainties, have the highest Bayesian evidence value before the potential correction. At the end of the section, we discuss the differences in the potential reconstruction between the various PSF, dust, and lens galaxies' light models.

To reconstruct the lens potential of $\mathrm{B} 1608+656$, we use a $130 \times 130$ pixel cutout of the drizzled ACS/F814W image with the pixel size 0'.03 shown in Figure 3. The galaxy-subtracted F814W image $(=\boldsymbol{d}-\mathbf{B} \cdot \mathbf{K} \cdot l)$ is a $130 \times 130$ subimage of the right-hand panel in Figure 8 with $200 \times 200$ pixels.

We follow the potential reconstruction method that was shown to succeed in Section 3. For the initial lens potential model, we use the SPLE1+D (isotropic) model from Koopmans et al. (2003). We perform nine iterations (labeled as 0-8) of pixelated potential corrections on B1608+656. For each iteration, we first reconstruct the source intensity on a $32 \times 32$ grid with pixel sizes of $0{ }^{\prime} 022$. The source region is chosen so that it maps to a completely joined annulus on the image plane (so that we can determine the relative potential difference between images). As in Section 5.6, the PSF is reduced to a $15 \times 15$ matrix to keep the inversion matrices sparse (and computation time low). Furthermore, we use only the curvature type of regularization for the source reconstruction to reduce computation time and to have regularized source-intensity gradients for the potential corrections. The source inversions are over-regularized in the early iterations to ensure a smooth resulting source for taking gradients. The source over-regularization factors start at 1000 and are gradually decreased to 1 at iteration $=8$. With the resulting source-intensity gradients and intensity deficits from the source reconstruction, we perform the potential correction on a grid of $30 \times 30$ pixels. We use the curvature form of regularization for each potential correction iteration. To keep the corrections linear, the potential corrections are also overregularized with the regularization constant $(\mu)$ set at 10 times the value where $\mu E_{\delta \psi}$ peaks, as in Section 3. The corrected potential has the midpoints in the left, bottom, and right parts of the annular reconstruction region fixed to the initial potential model.

The top row of Figure 10 shows the results of iteration $=0$ of source and potential reconstruction. The left-hand panel shows the reconstructed source that has been over-regularized by a factor of 1000. The caustics are those of the initial SPLE1+D (isotropic) model. The source is localized and compact, a sign that the initial SPLE1+D (isotropic) potential we started from is close to the true model. The middle-left panel shows significant image residuals that are to be corrected, especially near the cores of the images due to the over-regularization of the sourceintensity distribution. The annular region marks the region of data that we use for the evidence computation in the final iteration of source reconstruction. Using the gradient from the reconstructed source and the intensity deficit, the middle-right panel shows the potential reconstruction of iteration $=0$ and the right-hand panel shows the fraction of the accumulated potential corrections relative to the initial model.

The middle row of Figure 10 shows the result of iteration $=2$ of source and potential reconstruction. Compared to iteration $=$ 0 that has the same over-regularization factors, the source reconstruction is slightly smoother, the image residual has decreased, and the potential correction is not as large.

In the iterations from 3 to 8 , the potential corrections are small; therefore, the source reconstruction and image residual change only gradually during these iterations. The bottom row of Figure 10 shows the results of iteration $=8$ (the last iteration). The reconstructed source in the left-hand panel has more background noise than iteration $=2$ because the source is now optimally regularized. The source after the potential correction is more localized than that before the potential correction in Figure 9, which is a good indication that the reconstructed potential is closer to the true potential (up to the mass-sheet degeneracy). The normalized image residual in the middle left panel shows an overall decrease in the image residual compared with that in Figure 9. There remains intensity deficit near the image locations since the intensities of point-like images do not generally match due to the time delays and variability. This misfit can also be due to the undersampling of the PSF. There is also remaining image residual near image $\mathrm{C}$ that is likely due to imperfections in the dust correction. Nonetheless, the reduced $\chi^{2}$ inside the annulus is 1.1 (keeping in mind the unscaled nature of our image pixel uncertainties). The right-hand panel in the bottom row of Figure 10 shows that the final accumulated potential correction relative to the initial model is only $\sim 2 \%$. 


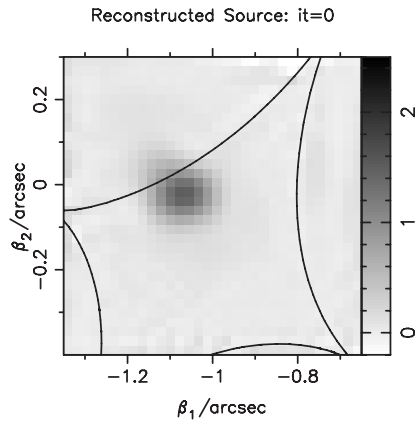

Reconstructed Source: it=2

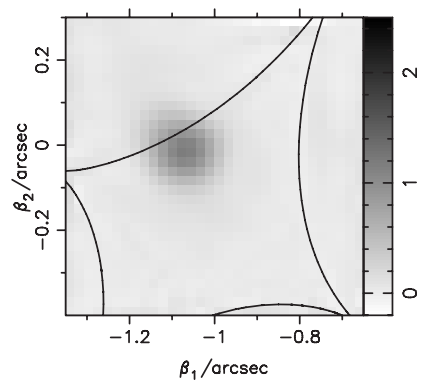

Reconstructed Source: it $=8$

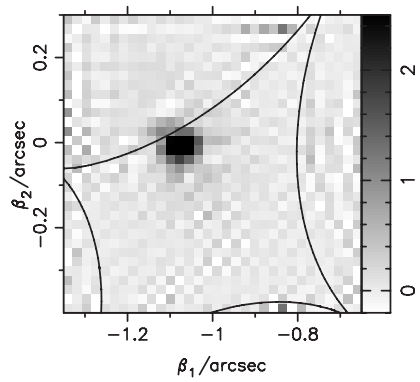

Normalized Image Res: it $=0$

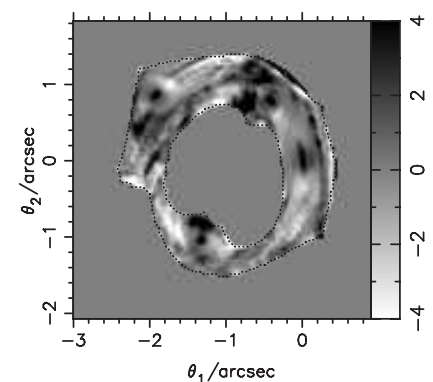

Normalized Image Res: it $=2$

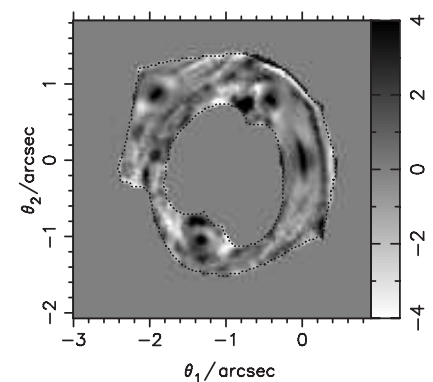

Normalized Image Res: it $=8$

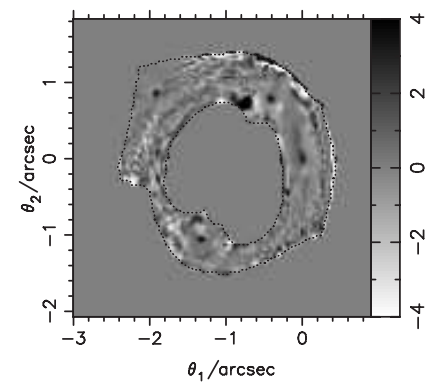

Potential Correction: it $=0$

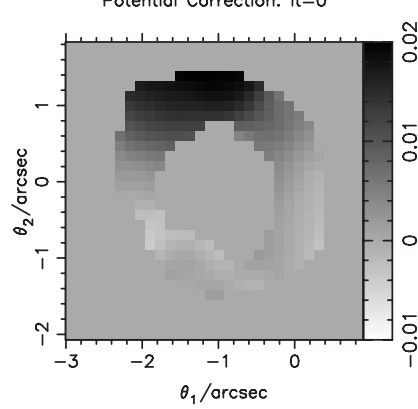

Potential Correction: $i t=2$

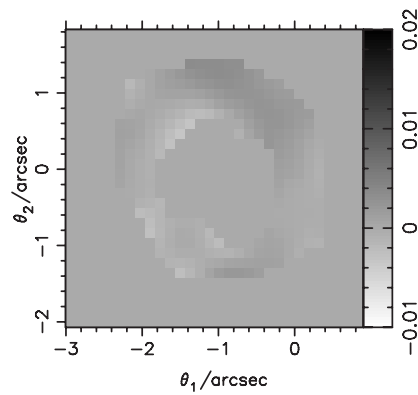

Potential Correction: it $=8$

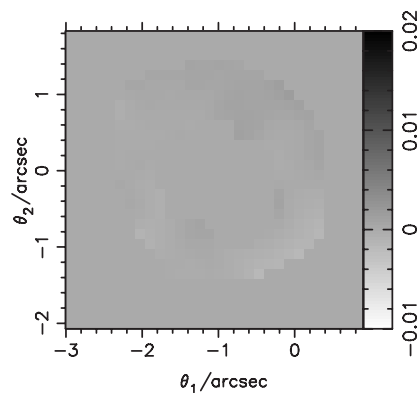

Frac of Accum Pot Corr: it $=0$

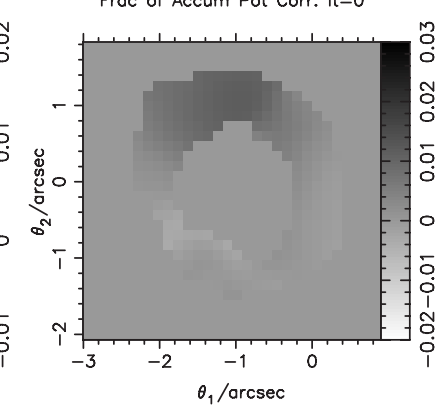

Frac of Accum Pot Corr: it $=2$

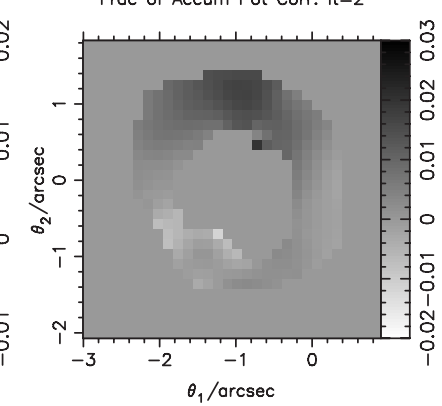

Frac of Accum Pot Corr: it $=8$

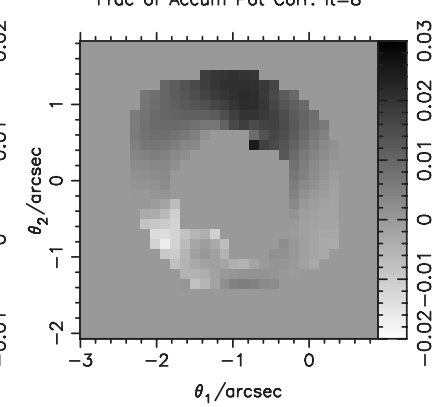

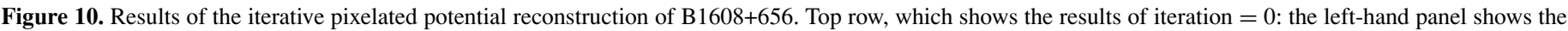

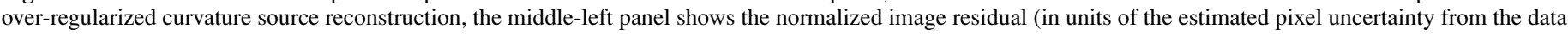

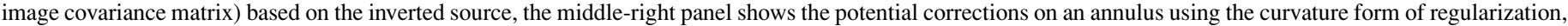

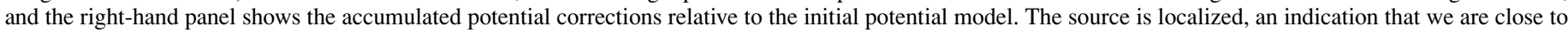

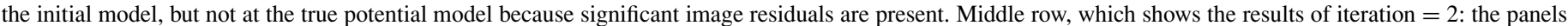

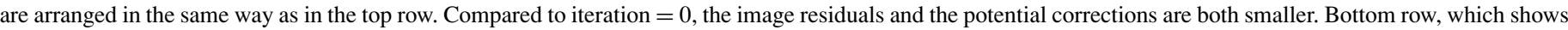

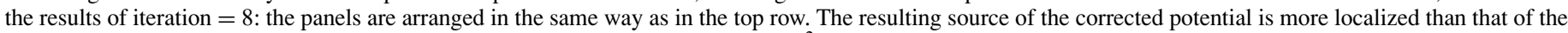
uncorrected potential in Figure 9, and the image residual corresponds to a reduced $\chi^{2}$ of 1.1 . The accumulated potential correction is only $\sim 2 \%$.

The structure of the accumulated potential correction may seem to resemble the simulation in Figure 1; however, this does not mean that the potential correction in B1608+656 corresponds to a mass clump as in the simulation. We point out that the maps of the potential corrections that generally look similar (due to the fixing of the three points in the annulus) may lead to very different convergence maps.

The potential reconstruction described above is for Model 5. After repeating the procedure for the other models, we find that the image residual and source reconstruction in the final iteration for the other three-band dust models are similar in feature to Model 5. In contrast, the two-band dust maps' source reconstruction continue to show nonlocalized source intensities with spurious light pixels outside of the main component. Furthermore, parts of the artificial surpluses or deficits of the dust-corrected light near the color boundaries remain after the potential correction. For Model 11 with no dust, the potential corrections lead to a localized source with image residuals that show misfit only near the image cores and locations of the dust lane.
These results of the potential reconstructions can be quantified using the Bayesian evidence values from the source reconstruction of the final corrected potential. Table 6 lists the evidence values for Models 2 to 11 . The uncertainties in the evidence values are due to the source pixelization and the possible range of over-regularization for the source-intensity reconstruction and lens potential correction. We explored over-regularization factors in the range between 1 and 1000 for the source intensity, and various factors within 30 of the regularization constant $\mu$ that corresponds to the peak of $\mu E_{\delta \psi}$ for the potential correction. The table shows that the three-band dust maps are consistently ranked higher than the two-band dust maps, indicating the importance of including the NICMOS image for the dust correction. All three-band dust maps give the same evidence values within the uncertainties, indicating that the various PSF and three-band dust models are all acceptable. Furthermore, the resulting Fermat potential differences between the images for these models agree within the uncertainties. Model 11 with no dust leads to the same evidence value as the values for three-band dust models. The predicted Fermat 
Table 6

Ranked Model Comparison after Potential Reconstruction

\begin{tabular}{rccc}
\hline \hline Model & PSF & Dust & $\begin{array}{c}\text { Log Evidence } \\
\left(\times 10^{4}\right)\end{array}$ \\
\hline 5 & B1 & Three-band & $1.77 \pm 0.05$ \\
9 & C & B1/three-band & $1.76 \pm 0.04$ \\
3 & C & Three-band & $1.76 \pm 0.05$ \\
11 & B1 & $\ldots$ & $1.76 \pm 0.05$ \\
2 & drz & Three-band & $1.75 \pm 0.05$ \\
7 & B2 & Three-band & $1.75 \pm 0.05$ \\
10 & B1 & C/two-band & $1.61 \pm 0.05$ \\
4 & C & Two-band & $1.58 \pm 0.05$ \\
6 & B1 & Two-band & $1.41 \pm 0.05$ \\
8 & B2 & Two-band & $1.40 \pm 0.05$ \\
\hline
\end{tabular}

Notes. In the PSF column, "drz" = drizzled TinyTim, "C" = closest star, "B1" = bright star \#1, and "B2" = bright star \#2. In the dust map column, "two-band" represents the dust map obtained from just the two ACS bands, and "three-band" represents the dust map obtained from the two ACS and the one NICMOS bands. The uncertainty in the log evidence from the source-intensity reconstruction is due to the source pixelization and the possible range of over-regularization for the source-intensity reconstruction and lens potential correction. Within the uncertainties, Models 5, 9, 3, 11, 2, and 7 have the highest evidence values. Note that the three-band dust maps are ranked higher than the two-band dust maps.

potential differences between the images for Model 11 are also in similar ranges as those of the three-band dust models. This shows that the global structure of the lens potential remains relatively intact after the dust correction to give similar predicted Fermat potential values, even though local pixelated potential corrections are flexible enough to mimic the effects of dust extinction. It is encouraging that the dust extinction in B1608+656 does not alter the surface brightness in a systematic way as to change the global structure of the lens potential. This robustness in the global structure of the lens potential is important for inferring the value of the Hubble constant.

In summary, for the top PSF, dust, and lens galaxies' light models, the pixelated potential correction scheme was successfully applied to B1608+656 leading to potential corrections of $\sim 2 \%$. This is only a small amount of correction, indicating that the smooth potential model in Koopmans et al. (2003) is remarkably good. The resulting source is also well localized.

This completes the dissection of the gravitational lens B1608+656. The image residual is not fully eliminated possibly due to imperfect PSF, dust, lens galaxies' light modeling, variability in the point source intensities, finite source resolution, and/or undersampled PSF. In Paper II, we use the models in Table 6 to derive $H_{0}$ and to estimate its uncertainty associated with the modeling.

\section{MASS AND LIGHT IN THE B1608+656 LENS SYSTEM}

The clean dissection of the lens system in the previous sections allows us to study the mass and light in G1 and G2.

Since the amount of potential correction is small, we can safely neglect the implied corrections when estimating the mass associated with the lens galaxies. Integrating the SPLE1+D (isotropic) surface mass density of each of the lens galaxies within their respective Einstein radii, the mass of $\mathrm{G} 1$ enclosed within $r_{\mathrm{E} ; \mathrm{G} 1}=0^{\prime} .81$ is $M_{\mathrm{G} 1}=1.9 \times 10^{11} h^{-1} M_{\odot}$ and the mass of G2 enclosed within $r_{\mathrm{E} ; \mathrm{G} 2}=0^{\prime} .28$ is $M_{\mathrm{G} 2}=2.8 \times 10^{10}$ $h^{-1} M_{\odot}$. Our dust correction enables us to recover the intrinsic luminosity of the lens galaxies. We use the fitted Sérsic light profiles to estimate the luminosity of G1 and G2. Integrating the flux of $\mathrm{G} 1$ and $\mathrm{G} 2$ within $r_{\mathrm{E} ; \mathrm{G} 1}$ and $r_{\mathrm{E} ; \mathrm{G} 2}$, respectively, the total mass to rest-frame $\mathrm{B}$-band light ratio of $\mathrm{G} 1$ is $\left(\mathrm{M} / L_{\mathrm{B}}\right)_{\mathrm{G} 1}=(2.0 \pm 0.2) h M_{\odot} L_{\mathrm{B}, \odot}^{-1}$ and of $\mathrm{G} 2$ is $\left(\mathrm{M} / L_{\mathrm{B}}\right)_{\mathrm{G} 2}=$ $(1.5 \pm 0.2) h M_{\odot} L_{\mathrm{B}, \odot}^{-1}$. The total mass and $\mathrm{M} / \mathrm{L}$ of $\mathrm{G} 1$ are consistent with those from earlier works on B1608+656 (e.g., Fassnacht et al. 1996) after taking into account the difference in the Einstein radius (due to the different number of components in the lens model) and the lowered $\mathrm{M} / \mathrm{L}$ as a result of the dust correction. The $\mathrm{M} / \mathrm{L}$ ratio of $\mathrm{G} 1$ is low compared to the lens galaxies in Treu \& Koopmans (2004), which have $\mathrm{M} / \mathrm{L}$ in the range $\sim 3-8 M_{\odot} / L_{\mathrm{B}, \odot}$. This is consistent with the spectrum of G1 showing signatures of both young and poststarburst populations, since these types of galaxies can have lower $\mathrm{M} / \mathrm{L}$ ratios by a factor of $\sim 10$ compared to other E/S0 galaxies at similar redshifts (e.g., van Dokkum \& Stanford 2003). Therefore, even though B1608+656 consists of two interacting galaxy lenses that lie in a group (Fassnacht et al. 2006), the $M / L$ ratio of $G 1$ is consistent with those in noninteracting lens systems.

\section{CONCLUSIONS}

In this paper, we have described and tested an iterative and perturbative lens potential reconstruction scheme whose accuracy in the recovered lens potential is in principle solely limited by the noise in the data, provided we have extended sources giving well connected ring-like images. The method is based on a Bayesian analysis, which provides a quantitative approach for comparing different models of the various constituents of a lens system: PSF, dust, lens galaxy light, and lens potential. We applied this method to the gravitational lens B1608+656 with deep HST ACS observations. We presented an image processing technique for obtaining a suite of PSF, dust, and lens galaxies' light models, and compared these models quantitatively. For each model, we reconstructed the lens potential on a grid of pixels, using the simply-parameterized SPLE1+D (isotropic) model in Koopmans et al. (2003) as our initial model. The reconstructions for the models with three-band dust maps were deemed successful in that they led to an acceptable level of image residual and a well-localized inferred source-intensity distribution.

From our analysis, we draw the following conclusions.

1. The potential reconstruction method, which simultaneously determines the extended source intensity and the lens potential distributions on grids of pixels, can correct for potential perturbations that are $\lesssim 5 \%$.

2. The mass-sheet degeneracy is broken in the potential corrections by choosing forms of regularization that suppress large deviations from the initial (mass-constrained) model unless the data require them.

3. The NICMOS F160W image is needed to complement the ACS F606W and F814W images for dust correction in order to avoid systematic errors.

4. The level of potential correction required in B1608+656 was found to be $\sim 2 \%$, validating the use of the simplyparameterized model of Koopmans et al. (2003).

5. The effect of dust extinction does not alter the global structure of the lens potential, and hence the predicted Fermat potential differences between the images.

6. The mass and $\mathrm{M} / L_{\mathrm{B}}$ of $\mathrm{G} 1$ inside $r_{\mathrm{E}}=0^{\prime \prime} .81$ are $1.9 \times 10^{11}$ $h^{-1} M_{\odot}$ and $(2.0 \pm 0.2) h M_{\odot} L_{\mathrm{B}, \odot}^{-1}$, respectively. These 
values are consistent with the spectral type of this galaxy and previous less accurate estimates of its $\mathrm{M} / \mathrm{L}$ ratio.

Although the pixelated potential reconstruction method can be applied to any lens system with an extended source-intensity distribution, it is particularly useful for measuring $H_{0}$ in timedelay lenses. B1608+656 is the only four-image gravitational lens system that have all three independent relative time delays measured with errors of a few percent (Fassnacht et al. 1999, 2002). However, current and future imaging surveys (such as the Canada-France-Hawaii Telescope (CFHT) Legacy Survey, the Panoramic Survey Telescope \& Rapid Response System, the Large Synoptic Survey Telescope, and the Joint Dark Energy Mission) either are or soon will be producing many more lenses: we can anticipate building up a sample of lens systems that can be fruitfully studied using the methods we have developed.

We thank M. Bradač, J. Krist, R. Massey, C. Peng, J. Rhodes, and P. Schneider for useful discussions and the anonymous referee for helpful comments that improved the presentation of the paper. We are grateful to M. Bradač and T. Schrabback for their help with the image processing. S.H.S. thanks the Kavli Institute for Theoretical Physics for the Graduate Fellowship in the fall of 2006 and for hosting the gravitational lensing workshop, during which significant progress on this work was made. S.H.S. acknowledges the support of the NSERC (Canada) through the Postgraduate Scholarship. C.D.F. and J.P.M. acknowledge support under the HST program \#GO-10158. Support for program \#GO-10158 was provided by NASA through a grant from the Space Telescope Science Institute, which is operated by the Association of Universities for Research in Astronomy, Inc., under NASA contract NAS 5-26555. C.D.F. and J.P.M. acknowledge the support from the European Community's Sixth Framework Marie Curie Research Training Network Programme, contract no. MRTN-CT-2004-505183 "ANGLES." L.V.E.K. is supported in part through an NWOVIDI career grant (project number 639.042.505). T.T. acknowledges support from the NSF through CAREER award NSF0642621, by the Sloan Foundation through a Sloan Research Fellowship, and by the Packard Foundation through a Packard Fellowship. This work was supported in part by the NSF under award AST-0444059, the Deutsche Forschungsgemeinschaft under the project SCHN 342/7-1 (S.H.S), the TABASGO foundation in the form of a research fellowship (P.J.M.), and by the US Department of Energy under contract number DEAC02-76SF00515. Based in part on observations made with the NASA/ESA Hubble Space Telescope, obtained at the Space Telescope Science Institute, which is operated by the Association of Universities for Research in Astronomy, Inc., under NASA contract NAS 5-26555. These observations are associated with program \#GO-10158.

\section{APPENDIX}

\section{THE MATRIX OPERATOR FOR PIXELATED POTENTIAL CORRECTION}

A comparison of the potential correction Equation (3) with its matrix form in Equation (4) shows that the matrix operator t needs to include the PSF blurring, the reconstructed sourceintensity gradient, and the gradient operator that acts on the potential perturbations $\delta \boldsymbol{\psi}$. We will consider each of these in the reverse order.

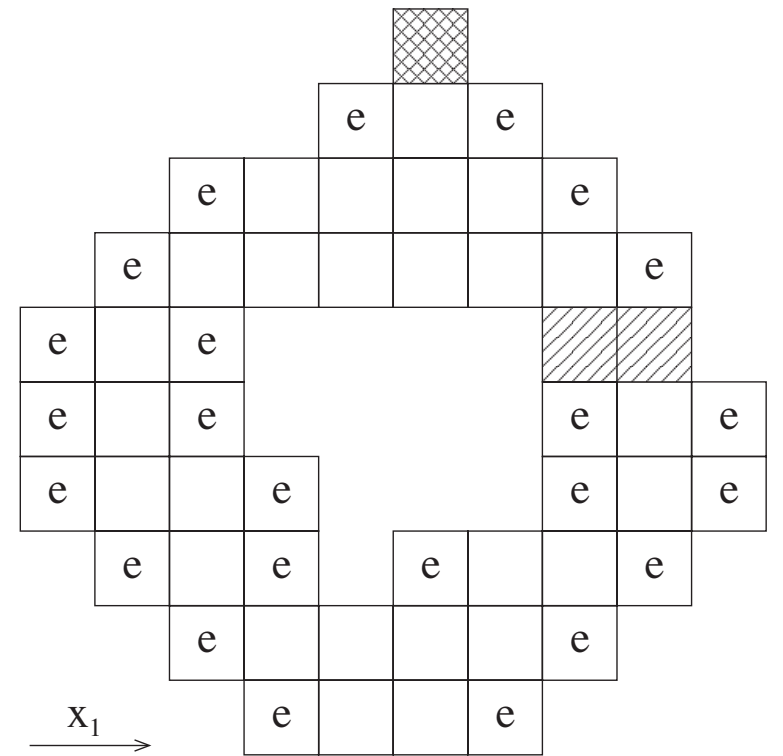

Figure 11. Typical annular region for potential corrections and the form of $\partial f_{i, j} / \partial x_{1}$ for each pixel. The blank pixels use the $i=2, \ldots, M-1$ expression for $\partial f_{i, j} / \partial x_{1}$ in equation (A1). The pixels with "e" are edge pixels that use the $i=1$ or $i=M$ expressions for $\partial f_{i, j} / \partial x_{1}$ in Equation (A1). The shaded pixels use Equation (A2) for $\partial f_{i, j} / \partial x_{1}$. The hashed pixel is an example of an "exposed" pixel with no adjacent pixel in the $x_{1}$ direction.

Before discussing the gradient operator, we need to define the domain over which the gradient operates. Recall that the potential corrections are obtained on an annular region that contains the Einstein ring of the lensed source. This region was obtained by tracing all the potential pixels back to the source plane (from the lens equation) and seeing which ones land on the finite source region of reconstruction. Only these potential pixels that trace back to the finite source region will have values of the source-intensity gradient for potential correction via Equation (3). These pixels tend to mark an annular region. Therefore, we need to find the gradient operator on this annular region for $\delta \psi$.

To construct the gradient operator, we use finite differencing to obtain numerical derivatives. For simplicity, first consider a $M \times N$ rectangular grid with $x_{1}$ and $x_{2}$ as axes and $(i, j)$ as pixel indices (typically $M \sim N \sim 30$ ). In this case, the partial derivatives of a function $f_{i, j}$ defined on the grid are

$\frac{\partial f_{i, j}}{\partial x_{1}}= \begin{cases}\frac{1}{2 \Delta x_{1}}\left(-3 f_{1, j}+4 f_{2, j}-f_{3, j}\right) & \text { if } i=1 \\ \frac{1}{2 \Delta x_{1}}\left(f_{i+1, j}-f_{i-1, j}\right) & \text { if } i=2, \ldots, M-1 \\ \frac{1}{2 \Delta x_{1}}\left(f_{M-2, j}-4 f_{M-1, j}+3 f_{M, j}\right) & \text { if } i=M\end{cases}$
$\frac{\partial f_{i, j}}{\partial x_{2}}= \begin{cases}\frac{1}{2 \Delta x_{2}}\left(-3 f_{i, 1}+4 f_{i, 2}-f_{i, 3}\right) & \text { if } j=1 \\ \frac{1}{2 \Delta x_{2}}\left(f_{i, j+1}-f_{i, j-1}\right) & \text { if } j=2, \ldots, N-1, \\ \frac{1}{2 \Delta x_{2}}\left(f_{i, N-2}-4 f_{i, N-1}+3 f_{i, N}\right) & \text { if } j=N\end{cases}$

where $\Delta x_{1}$ and $\Delta x_{2}$ are, respectively, the pixel sizes in the $x_{1}$ and $x_{2}$ directions. For the annular region of potential corrections, we only need to elaborate slightly on Equation (A1). Figure 11 shows a typical annular region and the types of pixels when numerically differentiating in the $x_{1}$ direction. The edge pixels of the annulus, which are denoted by "e" in the figure for the $x_{1}$ direction, are treated as though they are like the edge pixels of the rectangular grid (so that the $i=1, i=M, j=1$, or $j=N$ expressions are used) when the edge pixels are adjacent to at 
least two other pixels in the annulus in the direction of which the numerical derivative is taken. If an edge pixel of the annulus is only adjacent to one other pixel in the direction of which the numerical derivative is taken, such as the shaded pixels in the figure for the $x_{1}$ direction, then we construct the gradient by taking the difference between the two and dividing by the pixel size. For example, if $f_{i, j}$ is at the edge, and $f_{i+1, j}$ is also in the annulus (which will have to be an edge pixel if $f_{i+2, j}$ is not in the annulus), then the numerical derivatives in the $x_{1}$ direction for both $f_{i, j}$ and $f_{i+1, j}$ are

$$
\frac{\partial f_{i, j}}{\partial x_{1}}=\frac{f_{i+1, j}-f_{i, j}}{\Delta x_{1}} .
$$

A similar equation applies for the $x_{2}$ direction. If an edge pixel in the annulus is "exposed" in the sense that in one of the directions $x_{1}$ or $x_{2}$, it has no adjacent pixels in the annulus, then this pixel is removed from the annular region of reconstruction as no numerical derivative can be formed. An example of an "exposed" pixel in the $x_{1}$ direction is the hashed pixel in the figure. Following the above prescription, we can obtain the values $\left(\partial f_{i, j} / \partial x_{1}, \partial f_{i, j} / \partial x_{2}\right)$ of all the $(i, j)$ pixels in the annulus in terms of values of the function in the annulus $f_{k l}$. Factoring out the $f_{k l}$ values, we obtain the gradient operator defined as two matrices: $\mathbf{D}_{1}$ for $\partial / \partial x_{1}$ and $\mathbf{D}_{2}$ for $\partial / \partial x_{2}$.

To conform to the data grid (since the image residual and image covariance matrix is defined on the data grid), we use bilinear interpolation. We overlay the data grid on the coarser grid, and for every data pixel that lies inside the annular region on the coarse grid, we bilinearly interpolate to get, effectively, gradient operators on the data grid. This gives us an $N_{\mathrm{d}} \times N_{\mathrm{p}}$ matrix $\mathbf{G}$ where each row (corresponding to a data pixel that lies within the annulus) has four nonzero values that correspond to the coefficients of bilinearly interpolating among the four coarse potential pixels surrounding this data pixel. Associated with each data pixel are the source-intensity gradient values $\left(\partial I / \partial \beta_{1}\right.$ and $\partial I / \partial \beta_{2}$ ) that were obtained by mapping the data pixel back to the source plane using the lens equation, and interpolating on the reconstructed source-intensity gradient on the source grid. We define matrices $\mathbf{G}_{1}$ and $\mathbf{G}_{2}$ as the matrix $\mathbf{G}$ multiplied by the source-intensity gradient components $\partial I / \partial \beta_{1}$ and $\partial I / \partial \beta_{2}$, respectively. By definition, $\mathbf{G}_{1}$ and $\mathbf{G}_{2}$ are also $N_{\mathrm{d}} \times N_{\mathrm{p}}$ matrices.

Lastly, we represent the PSF as a blurring matrix (operator) B that is of dimensions $N_{\mathrm{d}} \times N_{\mathrm{d}}$ (see e.g., Section 4; Treu \& Koopmans 2004). Note that this matrix B is different from the matrix in Section 2.2.1 that is the Hessian of the $E_{\mathrm{D}}$.

Combining all the pieces together, the matrix operator $\mathbf{t}$ is

$$
\mathbf{t}=\mathbf{B} \cdot \mathbf{G}_{1} \cdot \mathbf{D}_{1}+\mathbf{B} \cdot \mathbf{G}_{2} \cdot \mathbf{D}_{2},
$$

which is of dimensions $N_{\mathrm{d}} \times N_{\mathrm{p}}$.

For the gravitational lens system B1608+656, we also need to include the effects of dust extinction, which we express as a diagonal matrix K. Tracing back along the light rays, we encounter the dust immediately after the PSF blurring (for the light from the lensed source). Therefore, we include it in Equation (A3) after B to get the following expression for the matrix operator $\mathbf{t}$ that includes dust:

$$
\mathbf{t}=\mathbf{B} \cdot \mathbf{K} \cdot \mathbf{G}_{1} \cdot \mathbf{D}_{1}+\mathbf{B} \cdot \mathbf{K} \cdot \mathbf{G}_{2} \cdot \mathbf{D}_{2}
$$

\section{REFERENCES}

Blandford, R., Surpi, G., \& Kundić, T. 2001, in ASP Conf. Ser. 237, Gravitational Lensing: Recent Progress and Future Goals, ed. T. G. Brainerd \& C. S Kochanek (San Francisco, CA: ASP), 65

Bradač, M., Schneider, P., Lombardi, M., \& Erben, T. 2005, A\&A, 437, 39

Brewer, B. J., \& Lewis, G. F. 2006, ApJ, 637, 608

Cardelli, J. A., Clayton, G. C., \& Mathis, J. S. 1989, ApJ, 345, 245

Casertano, S., et al. 2000, AJ, 120, 2747

Coleman, G. D., Wu, C.-C., \& Weedman, D. W. 1980, ApJS, 43, 393

Dressler, A., \& Gunn, J. E. 1983, ApJ, 270, 7

Dye, S., Evans, N. W., Belokurov, V., Warren, S. J., \& Hewitt, P. 2008, MNRAS, 388,384

Dye, S., \& Warren, S. J. 2005, ApJ, 623, 31

Falco, E. E., Gorenstein, M. V., \& Shapiro, I. I. 1985, ApJ, 289, L1

Fassnacht, C. D., Gal, R. R., Lubin, L. M., McKean, J. P., Squires, G. K., \& Readhead, A. C. S. 2006, ApJ, 642, 30

Fassnacht, C. D., Pearson, T. J., Readhead, A. C. S., Browne, I. W. A., Koopmans, L. V. E., Myers, S. T., \& Wilkinson, P. N. 1999, ApJ, 527, 498

Fassnacht, C. D., Womble, D. S., Neugebauer, G., Browne, I. W. A., Readhead, A. C. S., Matthews, K., \& Pearson, T. J. 1996, ApJ, 460, L103

Fassnacht, C. D., Xanthopoulos, E., Koopmans, L. V. E., \& Rusin, D. 2002, ApJ, 581,823

Hollis, J. M., Dorband, J. E., \& Yusef-Zadeh, F. 1992, ApJ, 386, 293

Hu, W. 2005, in ASP Conf. Series 339, Observing Dark Energy, ed. S. C. Wolff \& T. R. Lauer (San Francisco, CA: ASP), 215

Jee, M. J., et al. 2007, ApJ, 661, 728

Jullo, E., Kneib, J.-P., Limousin, M., Elíasdóttir, Á., Marshall, P. J., \& Verdugo, T. 2007, New J. Phys., 9, 447

Keeton, C. R. 2001, arXiv:astro-ph/0102340

Kneib, J.-P., Ellis, R. S., Smail, I., Couch, W. J., \& Sharples, R. M. 1996, ApJ, 471,643

Kochanek, C., Schneider, P., \& Wambsganss, J. 2006, Gravitational Lensing: Strong, Weak and Micro (New York: Springer)

Kochanek, C. S. 1991, ApJ, 373, 354

Koekemoer, A. M., Fruchter, A. S., Hook, R. N., \& Hack, W. 2002, in Proc. Workshop held at the Space Telescope Science Institute, The 2002 HST Calibration Workshop: Hubble after the Installation of the ACS and the NICMOS Cooling System, ed. S. Arribas, A. Koekemoer, \& B. Whitmore (Baltimore, MD: STScI), 337

Komatsu, E., et al. 2008, arXiv:0803.0547

Koopmans, L. V. E. 2005, MNRAS, 363, 1136

Koopmans, L. V. E., \& Fassnacht, C. D. 1999, ApJ, 527, 513

Koopmans, L. V. E., Treu, T., Fassnacht, C. D., Blandford, R. D., \& Surpi, G. 2003, ApJ, 599, 70

Kormann, R., Schneider, P., \& Bartelmann, M. 1994, A\&A, 284, 285

Krist, J. E., \& Hook, R. N. 1997, in The 1997 HST Calibration Workshop with a New Generation of Instruments, ed. S. Casertano, R. Jedrzejewski, T. Keyes, \& M. Stevens (Baltimore, MD: STScI), 192

MacKay, D. J. C. 1992, Neural Comput., 4, 415

Marshall, P. 2006, MNRAS, 372, 1289

Myers, S. T., et al. 1995, ApJ, 447, L5

Pei, Y. C. 1992, ApJ, 395, 130

Peng, C. Y., Ho, L. C., Impey, C. D., \& Rix, H.-W. 2002, AJ, 124, 266

Rhodes, J. D., et al. 2007, ApJS, 172, 203

Saha, P., Coles, J., Macciò, A. V., \& Williams, L. L. R. 2006, ApJ, 650, L17

Sérsic, J. L. 1968, Atlas de Galaxias Australes (Cordoba: Observatorio Astronomico)

Surpi, G., \& Blandford, R. D. 2003, ApJ, 584, 100

Suyu, S. H., \& Blandford, R. D. 2006, MNRAS, 366, 39

Suyu, S. H., Marshall, P. J., Hobson, M. P., \& Blandford, R. D. 2006, MNRAS, 371,983

Treu, T., \& Koopmans, L. V. E. 2004, ApJ, 611, 739

van Dokkum, P. G., \& Stanford, S. A. 2003, ApJ, 585, 78

Vegetti, S., \& Koopmans, L. V. E. 2008, arXiv:0805.0201

Wallington, S., Kochanek, C. S., \& Narayan, R. 1996, ApJ, 465, 64

Warren, S. J., \& Dye, S. 2003, ApJ, 590, 673

Wayth, R. B., \& Webster, R. L. 2006, MNRAS, 372, 1187

Williams, L. L. R., \& Saha, P. 2000, AJ, 119, 439

York, T., Jackson, N., Browne, I. W. A., Wucknitz, O., \& Skelton, J. E. 2005, MNRAS, 357, 124 